\title{
Depletion of GGA1 and GGA3 Mediates Postinjury Elevation of BACE1
}

\author{
Kendall R. Walker, ${ }^{1}$ Eugene L. Kang, ${ }^{1}$ Michael J. Whalen, ${ }^{2}$ Yong Shen, ${ }^{3}$ and Giuseppina Tesco ${ }^{1}$ \\ ${ }^{1}$ Alzheimer's Disease Research Laboratory, Department of Neuroscience, Tufts University School of Medicine, Boston, Massachusetts $02111,{ }^{2}$ Neuroscience \\ Center and Department of Pediatrics, Massachusetts General Hospital, Harvard Medical School, Charlestown, Massachusetts, 02129, and ${ }^{3}$ Center for \\ Advanced Therapeutic Strategies for Brain Disorders (CATSBD), Roskamp Institute, Sarasota, Florida 34243
}

Traumatic brain injury (TBI) is one of the most robust environmental risk factors for Alzheimer's disease (AD). Compelling evidence is accumulating that a single event of TBI is associated with increased levels of A $\beta$. However, the underlying molecular mechanisms remain unknown. We report here that the BACE1 interacting protein, GGA3, is depleted while BACE1 levels increase in the acute phase after injury $(48 \mathrm{~h}$ ) in a mouse model of TBI. We further demonstrated the role of GGA3 in the regulation of BACE1 in vivo by showing that BACE1 levels are increased in the brain of GGA3-null mice. We next found that head trauma potentiates BACE1 elevation in GGA3-null mice in the acute phase after TBI, and discovered that GGA1, a GGA3 homolog, is a novel caspase- 3 substrate depleted at $48 \mathrm{~h}$ after TBI. Moreover, GGA1 silencing potentiates BACE1 elevation induced by GGA3 deletion in neurons in vitro, indicating that GGA1 and GGA3 synergistically regulate BACE1. Accordingly, we found that levels of both GGA1 and GGA3 are depleted while BACE1 levels are increased in a series of postmortem AD brains. Finally, we show that GGA3 haploinsufficiency results in sustained elevation of BACE1 and A $\beta$ levels while GGA1 levels are restored in the subacute phase (7 d) after injury. In conclusion, our data indicate that depletion of GGA1 and GGA3 engender a rapid and robust elevation of BACE1 in the acute phase after injury. However, the efficient disposal of the acutely accumulated BACE1 solely depends on GGA3 levels in the subacute phase of injury.

\section{Introduction}

Alzheimer's disease (AD) is a complex disease influenced by the actions of multiple genes, their interactions with each other, and with the environment (Reitz et al., 2011). Traumatic brain injury (TBI) is one of the most robust environmental risk factors for $\mathrm{AD}$. TBI has been suggested to accelerate the onset of $\mathrm{AD}$, and the severity of the injury positively correlates with increased risk (Jellinger, 2004). Compelling evidence is mounting that a single TBI event is associated with increased levels of $A \beta$ and amyloid deposition both in humans and animal models (Johnson et al., 2010). Experimental TBI in rodents has been reported to increase levels of BACE1 (Blasko et al., 2004; Loane et al., 2009), suggesting that BACE1 elevation may be responsible for increased $A \beta$ production following TBI. However, the molecular mechanisms responsible for this postinjury elevation of BACE1 remain unknown.

BACE1 is a stress-related protease that is also upregulated in AD brains (Cole and Vassar, 2008). We have shown that BACE1

Received Oct. 30, 2011; revised June 4, 2012; accepted June 8, 2012.

Author contributions: K.R.W., M.J.W., and G.T. designed research; K.R.W., E.L.K., and M.J.W. performed research; Y.S. contributed unpublished reagents/analytic tools; K.R.W. and G.T. analyzed data; K.R.W. and G.T. wrote the paper.

This work was supported by Award Number R01AG033016 (to G.T.) and 1R01AG025952 (to G.T.) from the National Institute on Aging, and a grant from Cure Alzheimer's Fund (to G.T.). We thank Dr. Rudy Tanzi for his useful comments in the preparation and editing of this manuscript.

The authors declare no conflicting financial interests.

Correspondence should be addressed to Dr. Giuseppina Tesco, Alzheimer's Disease Research Laboratory, Department of Neuroscience, Tufts University School of Medicine, 136 Harrison Avenue, St 328A, Boston, MA 02111. E-mail: Giuseppina.Tesco@Tufts.edu.

DOI:10.1523/JNEUROSCI.5491-11.2012

Copyright $\odot 2012$ the authors $\quad 0270-6474 / 12 / 3210423-15 \$ 15.00 / 0$ increases following cerebral ischemia in rodents, and proposed that caspase-mediated depletion of the BACE1-interacting molecule GGA3 is the underlying mechanism of BACE1 elevation. GGA3 depletion stabilizes BACE1 by impairing its sorting to lysosomes where it is normally degraded (Koh et al., 2005; Tesco et al., 2007; Kang et al., 2010). We also reported that levels of GGA3 are decreased and inversely correlated with BACE1 levels in postmortem AD brains (Tesco et al., 2007). Levels of the GGA3 homolog, GGA1, are also decreased in AD brains (Wahle et al., 2006). GGA1 overexpression has been shown to decrease $A \beta$ levels (von Arnim et al., 2006; Wahle et al., 2006), most likely due to the increased retrograde transport of BACE1 from the endosomes to the trans-Golgi network (Wahle et al., 2005). Accordingly, GGA1 RNAi-mediated downregulation results in increased $\mathrm{A} \beta$ (Wahle et al., 2006) and BACE1 accumulation in the endosomes (He et al., 2005).

Here, we report that GGA3 is depleted while BACE1 levels increase in the acute phase after injury in a mouse model of TBI. We confirmed the role of GGA3 in the regulation of BACE1 in vivo by showing that BACE1 levels are increased in the brain of GGA3-null mice. We then found that head trauma potentiates BACE1 elevation in GGA3-null mice concurrently with caspasemediated depletion of GGA1. Furthermore, GGA1 silencing potentiates BACE1 elevation induced by GGA3 deletion in neurons in vitro. Collectively, these data indicate that GGA3 and GGA1 cooperatively regulate BACE1 degradation. Accordingly, decreased levels of GGA1 but not GGA2 are associated with depletion of GGA3 and elevation of BACE1 in a series of postmortem AD brains. Finally, we show that GGA3 haploinsufficiency results 
in sustained elevation of BACE1 and $\mathrm{A} \beta$ production (while GGA1 levels are restored) in the subacute phase of injury. These findings indicate that depletion of GGA1 and GGA3 leads to a rapid and robust elevation of BACE1 in the acute phase after injury. However, the efficient disposal of the acutely accumulated BACE1 depends solely on GGA3 levels in the subacute phase after injury.

\section{Materials and Methods}

Antibodies. The monoclonal antibody m3.2 (rodent APP, $\operatorname{sAPP} \alpha, \beta$-CTF, $\mathrm{A} \beta$ ) and Ab14 (against PS1) were a generous gift from Dr. P. Mathews (Center for Dementia Research, Nathan Kline Institute, Orangeburg, NY) and Dr. S. Gandy (Alzheimer's Disease Research Center, Mount Sinai School of Medicine, New York, NY), respectively. Polyclonal antiGGA1 (H-215) was purchased from Santa Cruz Biotechnology, polyclonal anti-GGA1 was a generous gift from Dr. M. Robinson (Cambridge Institute for Medical Research, University of Cambridge, Cambridge, UK), monoclonal GGA3 (612310) was from BD Transduction Laboratories, polyclonal anti-GGA3 (4167) from Cell Signaling Technology, monoclonal anti-GGA2 antibody from BD Transduction Laboratories, monoclonal GAPDH (MAB374) from Millipore, polyclonal anti-BACE1 (PA1-757) from Thermo Scientific, polyclonal anti-BACE1 (D10E5), monoclonal anti-myc (9B11), and polyclonal anti-caspase-3 (9665) were from Cell Signaling Technology, polyclonal anti-APP CTF (A8717) from Sigma, monoclonal anti-NeuN (MAB377) from Millipore, polyclonal anti- $\beta$-galactosidase (559761) from MP Biomedicals, and monoclonal anti-GFAP (GA5) was from Millipore. Secondary anti-mouse IgG HRP was from Thermo Scientific, anti-rabbit Ig G HRP from GE Healthcare, and mouse IgG trueblot secondary from eBioscience.

Animals. Five to seven month old and 18-24-month-old $G g a 3^{-/-}$, $\mathrm{Gga3}^{+/-}$, and $\mathrm{Gga3}^{+/+}$mice of both sexes were used in these experiments. Mice were housed under standard conditions and food and water were available ad libitum. All animal experiments were performed with the approval of Tufts University and Massachusetts General Hospital Institutional Animal Care and Use Committees.

Generation of $\mathrm{Gga}^{-1-}$ mouse line. The strain was created by microinjection of E14Tg2a.4 from 129P2/OlaHsd embryonic stem (ES) cells generated by BayGenomics (see http://baygenomics.ucsf.edu). The gene-trap vectors used within BayGenomics contain a splice-acceptor sequence upstream of a reporter gene, $\beta$-geo (a fusion of $\beta$-galactosidase and neomycin phosphotransferase II). These vectors insert randomly into introns. Chimeric males were mated to C57BL/6J females (Jackson Laboratories) and the resulting heterozygous male was purchased. We have developed a PCR-based protocol to genotype the mice using three primers: forward 1: 5' GTACATTGCT CCAAAGGAATAAGGTTvTAACG ' 3 ; reverse 1: 5' CTCACTACTTGCTA AACACTAGCTGAATGTGC' 3 ; reverse 2: 5' GACAGTATCGGCCTCAGG AAGATCGCACTC ' 3.

Wild-type samples yield bands at $\sim 1300 \mathrm{bp}$, homozygous samples yield bands at $\sim 1800 \mathrm{bp}$, and heterozygous samples yield bands at both molecular weights.

We determined that the gene-trap vector was inserted at nucleotide 1173 of intron 1 of Gga3 mouse gene (NM_173048) by sequencing the PCR products.

To confirm that only one copy of the gene trap vector ( $\beta$-galactosidase and neomycin resistance insert) inserted into the Gga3 gene, the first 100 mice bred were also subjected to PCR analysis for the neomycin resistance gene using the following primers: forward: 5' CAAATGGCGATTACCGTTGA ' 3 ; reverse: 5' TGCCCAGTCATAGCCGAATA '3.

Cresyl violet staining. Paraformaldehyde-fixed frozen sections $(30 \mu \mathrm{m})$ were incubated in $0.1 \%$ cresyl violet acetate (Sigma) at $37^{\circ} \mathrm{C}$ for $30 \mathrm{~min}$. Stained sections were briefly rinsed in water and differentiated in $95 \%$ ethanol. Sections were dehydrated in ethanol and cleared in xylene (Sigma) before mounting in histomount (Invitrogen).

$\beta$-Galactosidase staining. Coronal $(30 \mu \mathrm{m})$ and longitudinal $(60 \mu \mathrm{m})$ paraformaldehyde-fixed frozen sections were stained overnight $(\mathrm{O} / \mathrm{N})$ at $37^{\circ} \mathrm{C}$ using $\beta$-galactosidase reporter gene staining kit (Sigma) as per manufacturer's instructions. Sections were mounted in GelMount aqueous mounting medium (EMS).
Immunohistochemistry. Paraformaldehyde-fixed frozen sections (30 $\mu \mathrm{m})$ from $\mathrm{Gga3}^{+/+}$and $\mathrm{Gga3}^{-/-}$mice were blocked for $1 \mathrm{~h}$ at room temperature (RT) in 5\% Goat serum. Blocked sections were incubated in anti- $\beta$ galactosidase antibody $(1: 10,000)$ in combination with either antiNeuN $(1: 100)$ or anti-GFAP $(1: 500)$ in blocking solution at $4^{\circ} \mathrm{C}$ overnight. Sections were washed three times in PBS followed by incubation in AlexaFluor 488 or 568 secondary antibodies for $2 \mathrm{~h}$ at RT. Sections were washed and nuclei stained with DAPI. Sections were mounted on gelatincoated slides with fluorescent mounting medium (Dako). Fluorescent tissue sections were imaged on a Nikon A1R confocal microscope with Plan Apo VC $20 \times$ (air) and 60× (oil immersion) objectives. Background fluorescent staining of the negative control tissue from $\mathrm{Gga}^{+/+}$mice was used to set baseline for the laser strength and gain for image capture of the $\beta$-galactosidase staining in $\mathrm{Gga3}^{-/-}$tissue sections. Z-stacks were captured in $2 \mu \mathrm{m}$ increments and analyzed in NIS elements software (Nikon).

Controlled cortical impact experimental TBI. Briefly, 6-month-old $\mathrm{Gga3}^{-/-}, \mathrm{Gga3}^{+/-}$, and $\mathrm{Gga3}^{+/+}$mice were anesthetized with $4 \%$ isoflurane (Anaquest) in $70 \% \mathrm{~N}_{2} \mathrm{O}$ and $30 \% \mathrm{O}_{2}$ using a Fluotec 3 vaporizer (Colonial Medical) and positioned in a stereotaxic frame. Anesthesia was maintained using $2 \%$ to $3 \%$ isoflurane $\mathrm{N}_{2} \mathrm{O} / \mathrm{O}_{2}$. Following a mid-line incision, a $5 \mathrm{~mm}$ craniotomy was made using a portable drill over the left parietotemporal cortex, and the bone flap was removed. Mice were then subjected to controlled cortical impact (CCI) using a pneumatic cylinder with a $3 \mathrm{~mm}$ flat-tip impounder, velocity $6 \mathrm{~m} / \mathrm{s}$, depth $0.6 \mathrm{~mm}$, and impact duration $100 \mathrm{~ms}$. Cotton swabs were used to absorb and control any bleeding after impact. The bone flap was discarded and the scalp was sutured closed. Mice were allowed to recover in their cage.

Lesion volume analysis. Two weeks after TBI, mice were unrecoverably sedated with isoflurane followed by perfusion by $4 \%$ paraformaldehyde. Brains were carefully removed and fixed overnight at $4^{\circ} \mathrm{C}$ in $4 \%$ paraformaldehyde, followed by cryopreservation in $30 \%$ sucrose for $3 \mathrm{~d}$ at $4^{\circ} \mathrm{C}$. Lesion volume analysis was performed as previously described (Wang et al., 2000). Briefly, cryopreserved brains were sectioned on a sliding microtome. Twenty-five micrometer sections were cut and every 20th section was collected $\left(500 \mu \mathrm{m}\right.$ intervals). Lesion volume $\left(\mathrm{mm}^{3}\right)$ was determined using MCID Analysis software by carefully tracing the area of the cavitary lesion in each collected section. Each lesion area was measured three times and the average was taken of the measurements.

Staurosporine-induced apoptosis of $\mathrm{H4}-751$ cells. H4-751 cells cultured in DMEM supplemented with $10 \%$ FBS and $200 \mu \mathrm{g} / \mathrm{ml} \mathrm{G418}$ were incubated in the presence of $1 \mu \mathrm{M}$ staurosporine (STS; Calbiochem) with or without $50 \mu \mathrm{M}$ of the general caspase inhibitor zVAD (Calbiochem) for $8 \mathrm{~h}$ at $37^{\circ} \mathrm{C}$. Cell lysates were subjected to electrophoresis on a $4-12 \%$ Bis-Tris acrylamide gel, and Western blotting was performed using an anti-GGAl antibody at a 1:1000 dilution (H-215, Santa Cruz Biotechnology).

In vitro translation of GGA3, GGA1, and D306AGGA1, site-directed mutagenesis, and recombinant caspase- 3 cleavage assay. The HA-GGA3 pcDNA4 plasmid was a generous gift from Dr. Waguri (Osaka University, Graduate School of Medicine, Osaka, Japan). The myc-GGA1 pCR3.1 plasmid was a generous gift from Dr. Juan Bonifacino (Cell Biology and Metabolism Program, Eunice Kennedy Shriver National Institute of Child Health and Human Development, NIH, Bethesda, MD). The D306AGGA1 plasmid was generated by site-directed mutagenesis of the my-GGA1 pCR3.1 plasmid. GGA3, GGA1, and D306AGGA1 were in vitro translated (IVT) in the presence of cold methionine using TNT Quick Coupled Transcription/Translation Systems as recommended by the manufacturer (Promega). GGA3 and GGA1 IVT reactions $(3.5 \mu \mathrm{l})$ were incubated with or without increasing amounts (200 through $600 \mathrm{ng}$ ) of recombinant caspase-3 (Pharmingen) in caspase reaction buffer at $37^{\circ} \mathrm{C}$ for $16 \mathrm{~h}$. GGA1 and D306AGGA1 IVT reactions $(3.5 \mu \mathrm{l})$ were incubated with or without $12 \mu \mathrm{M}$ recombinant caspase-3 for $16 \mathrm{~h}$ at $37^{\circ} \mathrm{C}$.

Site-directed mutagenesis. Site-directed mutagenesis was performed using the QuikChangeSite-Directed Mutagenesis Kit (Stratagene) according to manufacturer's instructions. Briefly, The D306A GGA1 plasmid was generated by PCR-based site-directed mutagenesis of the myc-GGA1 pCR3.1 plasmid 
using the following primers: 5' GGAGGTCAACGGTGCTGCCACAGCCGGC TC3'; 5' CCTCCAGTTGCCACGACGGTGTCGGCCGAG3'.

Preparation of naive tissue homogenates for immunoblotting and $\beta$-secretase activity assay. Frozen tissue (hemibrains or hippocampi) from 5-7-month-old and 18-24-month-old $\mathrm{Gga3}^{-/-}, \mathrm{Gga3}^{+/-}$, and $\mathrm{Gga3}^{+/+}$mice were homogenized in modified RIPA buffer for immunoblotting. Briefly, snap-frozen tissue from each mouse was homogenized in 10 volumes of modified RIPA buffer supplemented with protease and phosphatase inhibitors. The homogenate was then centrifuged at $20,000 \times g$ for $15 \mathrm{~min}$ at $4^{\circ} \mathrm{C}$. The supernatant was collected and further clarified by a second centrifugation. Protein concentration was determined using the BCA method (Thermo Scientific). Homogenates were divided into aliquots and stored at $-80^{\circ} \mathrm{C}$ until analysis.

$\beta$-Secretase activity assay. $\beta$-Secretase activity was measured in tissue homogenates using a highly sensitive FRET-based cleavage assay as described by Fukumoto et al. (2002) with modifications. Briefly, 96-well microplates were coated with anti-BACE1 antibody (D10E5, Cell Signaling Technology, 1:1000) at RT for $8 \mathrm{~h}$. Excess unbound antibody was removed by washing with PBS. The coated plate was blocked using $1 \% \mathrm{BSA} / \mathrm{PBS}$ overnight at $4^{\circ} \mathrm{C}$. RIPA lysed hemibrain homogenates from $\mathrm{Gga3}^{+/+}, \mathrm{Gga3}^{+/-}$, and $\mathrm{Gga3}^{-/-}$mice $(50-100 \mu \mathrm{g})$ were incubated for $1 \mathrm{~h}$ at $37^{\circ} \mathrm{C}$ followed by extensive washing with PBS. Bound BACE1 activity was measured by using $10 \mu \mathrm{M}$ fluorogenic $\beta$-secretase substrate IV (Millipore) in assay buffer (50 mm sodium acetate pH4.5, $10 \mathrm{~mm}$ $\mathrm{NaCl}, 0.002 \%$ Triton-X, $1 \mathrm{~mm}$ DTT) in the dark at $37^{\circ} \mathrm{C}$. The fluorescent signal resulting from cleavage of the substrate was measured at intervals over a $24 \mathrm{~h}$ time period using a Synergy 2 plate reader (excitation $340 \mathrm{~nm}$, emission $485 \mathrm{~nm}$ ) (Biotek). To control for nonspecific cleavage of the fluorogenic substrate, an equal amount of hemibrain lysate from a Bace1-null mouse was included in each assay.

Preparation of naive tissue homogenates for detection of endogenous levels of A $\beta X-40$ using a WAKOII rodent/human ELISA. Frozen tissue (Hemibrains or Hippocampi) from 5-7-month-old and 18-24-monthold $\mathrm{Gga3}^{-/-}, \mathrm{Gga3}^{+/-}$and $\mathrm{Gga3}^{+/+}$mice were homogenized in a diethylamine extraction buffer (DEA) for analysis of endogenous secreted $\mathrm{A} \beta$. Briefly, snap frozen tissue (hemibrain or hippocampus) from each mouse was homogenized in 10 volumes of chilled DEA extraction buffer (0.2\% DEA, $50 \mathrm{~mm} \mathrm{NaCl}, 2 \mathrm{~mm}$ PNT, $1 \mathrm{~mm}$ AEBSF, protease and phosphatase inhibitor cocktail) and centrifuged at $100,000 \times g$ for $1 \mathrm{~h}$ at $4^{\circ} \mathrm{C}$ in a Beckman Ultima Ultracentrifuge. The supernatant was collected and neutralized with $1 / 10$ th volume of $0.5 \mathrm{M}$ Tris- $\mathrm{HCl}$, $\mathrm{pH}$ 6.8. Protein concentrations were determined via the BCA method. Homogenates were frozen at $-80^{\circ} \mathrm{C}$ until ELISA analysis.

Preparation of CCI contusions for immunoblotting and $A \beta$ analysis. Six-month-old $\mathrm{Gga3}^{-/-}, \mathrm{Gga3}^{+/-}$, and $\mathrm{Gga}^{+/+}$mice subjected to experimental TBI were killed by isoflurane sedation followed by decapitation $48 \mathrm{~h}$ and $7 \mathrm{~d}$ after CCI. Ipsilateral contusions (cortex and hippocampus) were dissected and snap frozen in liquid nitrogen. The identical area in the contralateral (uninjured) hemisphere was also dissected and snap frozen in liquid nitrogen to serve as an internal control. The snap frozen tissues were homogenized in 10 volumes of modified RIPA buffer supplemented with protease inhibitors. The homogenate was then centrifuged at $20,000 \times g$ for $15 \mathrm{~min}$ at $4^{\circ} \mathrm{C}$. The supernatant was collected and further clarified by a second centrifugation. Protein concentration was determined using the BCA method (Thermo Scientific). Homogenates were divided into aliquots and stored at $-80^{\circ} \mathrm{C}$ until analysis.

Immunoblotting of proteins in Naive and TBI tissue lysates. RIPA extracted protein lysates $(15-50 \mu \mathrm{g})$ were electrophoresed on $4-12 \%$ BisTris NUPAGE gels (Invitrogen). Proteins were electroblotted onto PVDF (Bio-Rad) membrane and blocked in 5\% skim milk/TBST. Membranes were incubated in primary antibody $\mathrm{O} / \mathrm{N}$ at $4^{\circ} \mathrm{C}$, washed $3 \times$ in TBST, and incubated in secondary antibody, either anti-mouse-HRP or antirabbit-HRP (1:10,000 dilution), for $1 \mathrm{~h}$ at RT. Membranes were detected chemiluminescently using either ECL (Thermo Scientific), ECL-Plus (GE Healthcare), or Femto (Thermo Scientific) chemiluminescent reagents. Chemiluminescent signal was captured on an LAS4000 Fuji Imager.

Detection of endogenous $A \beta x-40$ using WAKOII Rodent/Human $x-40$ ELISA. Endogenous $\mathrm{A} \beta \mathrm{x}-40$ was detected in naive hemibrains and hip- pocampi (DEA soluble) and RIPA soluble CCI contusion extracts using the WAKOII rodent/human ELISA according manufacturer's instructions. The WAKOII rodent/human ELISA employs the well characterized BNT77/BA27 antibody system to detect A $\beta \mathrm{x}-40$ (Wako Chemicals). For detection of endogenous $A \beta 40$ in the naive hemibrains and hippocampi, $100 \mu \mathrm{l}$ of DEA extract was analyzed. RIPA extracts $(1-2.5 \mu \mathrm{g} /$ $\mu \mathrm{l})$ prepared from the CCI contusions were used to detect RIPA soluble $\mathrm{A} \beta 40$ in the injured and contralateral hemispheres of mice subjected to CCI.

Lentiviral packaging and infection of primary cortical neurons. Cortical neurons were extracted from postnatal day 1 (P1) mouse pups as described in the study by Ninan and Arancio (2004). Briefly, neocortex was dissected and digested with $0.25 \%$ trypsin at $37^{\circ} \mathrm{C}$ for $15 \mathrm{~min}$. Cells were cultured in Neurobasal A supplemented with 2\% B27, 1\% FBS, 0.4 mM L-Glut, $6.6 \mathrm{ng} / \mathrm{ml} 5$ fluorodeoxyuridine, and $16.4 \mathrm{ng} / \mathrm{ml}$ uridine. A $50 \%$ media change was performed every $4 \mathrm{~d}$. Mission shRNA plasmids expressing shRNAs against murine GGAl (TRCN0000115330) and a negative control (Sigma) were packaged into lentiviruses as described by Sena-Esteves et al. (2004). Lentiviruses were titered using the Quicktiter Lentivirus ELISA kit (Cell Biolabs). Cortical neurons were infected on DIV3 with lentiviruses expressing shRNA against murine GGA1 or a negative control at an multiplicity of infection (MOI) 5 for $6 \mathrm{~h}$ at $37^{\circ} \mathrm{C}$. After $6 \mathrm{~h}$, the virus was replaced with conditioned Neurobasal A media from cortical neurons not subject to viral infection.

Human brain samples. Twenty AD and 19 nondemented (ND) temporal cortex were obtained from the Brain Donation Program, Sun Health Research Institute, Sun City, Arizona. Human tissue was collected with informed consent of subjects or next of kin and with ethical approval from the Sun Health IRB. Protein lysates were prepared by homogenization of frozen temporal cortex in modified RIPA buffer supplemented with protease inhibitors (Thermo Scientific).

Densitometry and statistical analysis. Digital Images were collected using either a Versadoc (Bio-Rad) or LAS-4000 (Fuji) imager. Densitometry analysis was performed on a Macintosh computer using QuantityOne software (Bio-Rad). Statistical analysis was performed using Instat3 software (GraphPad Software Inc.). Unpaired or paired $t$ test was used for datasets that passed normality test. Unpaired $t$ test with Welch correction was used for datasets that passed a normality test but had different SDs. Mann-Whitney test was used for datasets that did not pass a normality test.

\section{Results}

\section{Levels of BACE1 and A $\beta$ increase while GGA3 is depleted following TBI}

Head trauma was induced by the CCI model as previously described (Bermpohl et al., 2006) in C57BL/6J mice. At various time intervals after CCI, mice were killed and brains collected. Brain homogenates were prepared from the ipsilateral/injured (I) and contralateral/control (C) hemispheres, and Western blot analysis was performed as previously described (Tesco et al., 2007). Following CCI, GGA3 was depleted while BACE1 increased (Fig. 1). Since APP is also a substrate for caspase cleavage (LeBlanc, 2005), we tested whether APP undergoes caspase-mediated cleavage in the mouse brain following TBI, and found that full-length APP protein levels were slightly decreased because of the generation of a $90 \mathrm{kDa}$ fragment previously reported to be the $\mathrm{N}$-terminal APP caspase fragment in cells undergoing apoptosis and in ischemic rat brain (Tesco et al., 2003, 2007). Thus, GGA3 depletion and elevated BACE1 levels occur concomitantly with caspase activation (Fig. 1).

Next, RIPA-soluble A $\beta 40$ levels were measured in contralateral (C) and injured (I) hemispheres of 6 C57BL/6J mice $48 \mathrm{~h}$ after injury using a commercial ELISA kit employing the well characterized BNT77/BA27 antibody system (Wako Chemicals). $\mathrm{A} \beta$ levels were increased by $\sim 50 \%(10.2 \pm 0.82$ vs $6.87 \pm 0.24$ $\mathrm{pmol} / \mathrm{g}$ protein, $p=0.0038$ ) in the injured hemisphere compared 


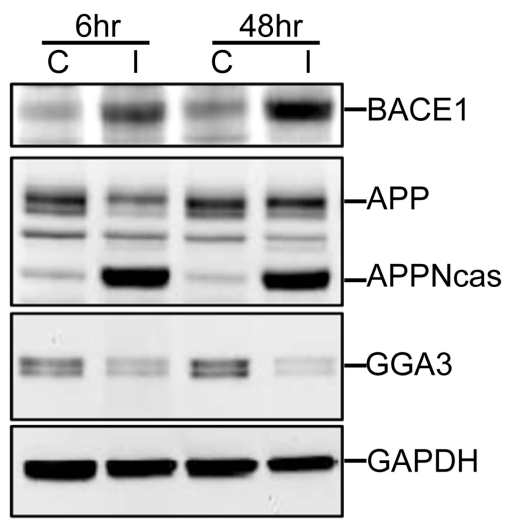

Figure 1. Levels of $B A C E 1$ and $A \beta$ increase while $G G A 3$ is depleted following TBI. Brains were collected after the indicated hours following injury. BACE1 (PA1-757), GGA3 (612310), APP (A8717), or GAPDH (MAB374) levels were detected by Western blot analysis in extracts obtained from contralateral (C) or injured (I) hemispheres. BACE1 was elevated in injured hemispheres. Caspase-mediated cleavage of full-length APP results in the production of an N-terminal caspase fragment (APPNcas) detected in injured hemisphere. GGA3 was depleted in injured hemispheres. GAPDH levels were unchanged and used as a loading control. BACE1 levels were normalized to GAPDH and expressed as arbitrary units (mean \pm SEM) of 6 C57BL/6J mice. BACE1 levels were elevated 1.6-fold in the injured hemisphere at $48 \mathrm{~h}$ after injury $(1.29 \pm 0.074$ vs $0.81+0.037, p=0.0003)$. Statistical analysis of data was performed using a paired $t$ test.

with the contralateral hemisphere. As a negative control, $\mathrm{A} \beta$ levels were also measured in the injured and contralateral hemisphere of $\mathrm{APP}^{-1-}$ mice (purchased from Jackson Laboratory) (data not shown). Our aim was to assess $\beta$-secretase activity, and since increases in BACE1 activity have previously been shown to increase both $A \beta 40$ and $A \beta 42$ (Vassar et al., 1999), we did not measure $\mathrm{A} \beta 42$ in these experiments. Collectively, these findings indicate that GGA3 depletion, mediated by caspase cleavage, and the consequent BACE1 elevation may be a common underlying mechanism of increased $\mathrm{A} \beta$ production following cerebral ischemia and TBI.

\section{Generation and characterization of GGA3-null mice}

To investigate GGA3 regulation of BACE1 in vivo we analyzed mice with heterozygous or homozygous deletions of the Gga3 gene $\left(\mathrm{Gga3}^{+/-}\right.$and $\mathrm{Gga3}^{-/-}$, respectively). $\mathrm{Gga3}^{+/-}$founder mice were generated by the Mutant Mouse Regional Resource Centers at University of California, Davis (MMRRC) using a gene-trapping method (see http://www.genetrap.org). The gene-trap vectors contain a splice-acceptor sequence upstream of a reporter gene, $\beta$-geo (a fusion of $\beta$-galactosidase and neomycin phosphotransferase II) (Stryke et al., 2003). We developed a three primer PCR-based protocol to genotype the mice (Fig. $2 A, B$ ) and determined that the gene-trap vector inserted at nucleotide 1173 of intron 1 of the Gga3 mouse gene (ENSMUST00000019135.916). Of the first 100 mice bred, every neomycin-positive mouse was also positive for the 1800 kb Gga3-null PCR product, confirming that this line has only one insertion of the gene trap construct. Intercrosses of $\mathrm{Gga}^{+/-}$mice produced $\mathrm{Gga}^{-/-}$mice in a normal Mendelian fashion that are healthy, viable, and fertile. Analysis of neural tissue from 6-monthold littermates revealed no gross anatomical defects in GGA3-null mice (Fig. 2C). $\beta$-Galactosidase staining of $\mathrm{Gga3}^{-/-}$mouse brain was used to determine the expression pattern of GGA3 in the adult mouse brain. GGA3 is ubiquitously expressed throughout the brain with the highest levels of expression in the hippocampus, cortex, and cerebellum (Fig. 2D). Confocal analysis of $\mathrm{Gga3}^{-/-}$tissue sections stained with an antibody against $\beta$-galactosidase revealed an expression pattern of GGA3 throughout the mouse brain identical to that seen with the enzymatic $\beta$-galactosidase staining (data not shown). Next, we determined that GGA3 is mainly expressed in neuronal cells by performing confocal microscopy analysis of brain sections costained with anti- $\beta$-galactosidase antibody and neuronal (NeuN) or glial (GFAP) markers. Colocalization studies were performed in the cortex, hippocampus (CA1, CA3, dentate gyrus), and midbrain. Costaining in the CA1 region of the hippocampus is shown as an example (Fig. 2E-J).

Western blot analysis using two different anti-GGA3 antibodies revealed that the GGA3 protein is absent in brain extracts from $\mathrm{Gga3}^{-/-}$mice while the levels are reduced by $\sim 50 \%$ in $\mathrm{Gga3}^{+/-}$ mice (Fig. $2 \mathrm{~K}$ ). Given that previous reports have shown that GGA1 levels are significantly decreased $(\sim 40 \%)$ in $\mathrm{AD}$ brains (Wahle et al., 2006) and that GGA1 overexpression decreases A $\beta$ levels (von Arnim et al., 2006; Wahle et al., 2006), we assessed the levels of GGA1 in GGA3-null mice and found that the genetic ablation of GGA3 does not produce a compensatory increase in GGA1 (Fig. $2 K, L$ ).

\section{Genetic deletion of GGA3 increases levels of BACE1 in vitro and in vivo}

BACE1 and $\mathrm{A} \beta$ levels were assessed in primary cortical neuronal cultures (DIV 8) from $\mathrm{Gga3}^{+/+}$and $\mathrm{Gga3}^{-/-} \mathrm{P} 1$ pups. Levels of BACE1 were found to be increased twofold $(p=0.0003)$ in DIV $8 \mathrm{Gga3}^{-/-}$cortical neurons compared with $\mathrm{Gga3}^{+/+}$neurons (Fig. $3 A, B$ ). Levels of GGA1, APP, and PS1 were unchanged (Fig. $3 A$ ). Consistent with the BACE1 elevation, $\mathrm{A} \beta \mathrm{x}-40$ levels were increased by $\sim 3$-fold $(p=0.0001)$ in $\mathrm{Gga3}^{-\prime-}$ compared with $\mathrm{Gga3}^{+/+}$cultures (Fig. $3 C$ ).

We measured BACE1 protein levels in 5-7-month-old $\mathrm{Gga3}^{+/+}, \mathrm{Gga3}^{+/-}$, and $\mathrm{Gga3}^{-/-}$mice of both sexes and found that BACE1 was increased $\sim 30 \%$ in the brains of $\mathrm{Gga3}^{-/-}$ mice compared with $\mathrm{Gga3}^{+/+}$littermate controls. BACE1 levels were comparable between $\mathrm{Gga3}^{+/-}$and $\mathrm{Gga}^{+/+}$mice (Fig. $3 D$ ). Further analysis revealed that the effect of the Gga3 genetic deletion is specific for BACE1 as there was no detectable change in SorLA, another GGA-binding protein (Rogaeva et al., 2007), PS1, the catalytic component of the $\gamma$-secretase complex, or APP levels between genotypes (Fig. 3E).

$\beta$-Secretase activity was assessed by two different methods: measuring $\beta$-CTF (C99) levels in brain extracts using the m3.2 antibody (kind gift from Paul Matthews) and a highly sensitive FRET-based $\beta$-secretase cleavage assay. We found no statistical difference in $\beta$-CTF (C99) levels between $\mathrm{Gga3}^{-/-}, \mathrm{Gga}^{+/-}$, and $\mathrm{Gga3}^{+/+}$littermate controls (Fig. 3F). Additionally, no statistical difference was observed in $\beta$-secretase activity between $\mathrm{Gga3}^{-1-}$, $\mathrm{Gga3}^{+/-}$, and $\mathrm{Gga3}^{+/+}$littermate controls as measured by cleavage of the fluorogenic $\beta$-secretase IV substrate (Fig. $3 G$ ). Accordingly, no difference was observed in $\mathrm{A} \beta \mathrm{x}-40$ levels between $\mathrm{Gga3}^{-/-}$and $\mathrm{Gga3}^{+/+}$mice (Fig. $3 \mathrm{H}$ ). The differing results obtained in vitro and in vivo could be attributed to the presence of non-neuronal cells in brain extracts when compared with neuronalenriched cultures. BACE1 expression is predominately neuronal (Vassar et al., 1999). As a consequence, BACE1 and A $\beta$ levels are robustly increased in $\mathrm{Gga}^{-/-}$primary neuronal cultures containing negligible amount of non-neuronal cells; whereas the presence of non-neuronal cells in brain extracts may mask the effect of GGA3 deletion on BACE1 and $\mathrm{A} \beta$, resulting in a smaller increase in BACE1 and a failure to increase $A \beta$ levels in the $\mathrm{Gga}^{-1-}$ brain. Another alternative explanation is suggested by previous reports showing that the effect of BACE1 overexpression or haploinsufficiency on $A \beta$ levels (endogenous or human transgenic) is minimal in young adult mice (Luo et al., 2001; McConlogue et al., 2007; Hirata-Fukae et al., 
A

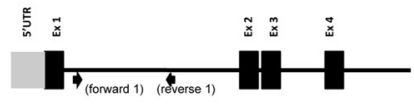

wild type Gga3 allele

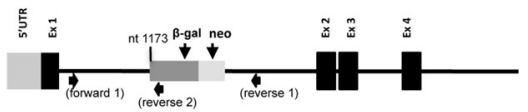

trapped Gga3 allele

C

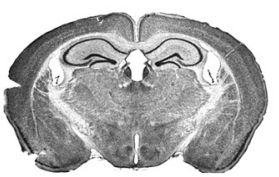

Gga3 -/-

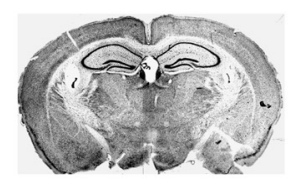

Gga3+/+

E

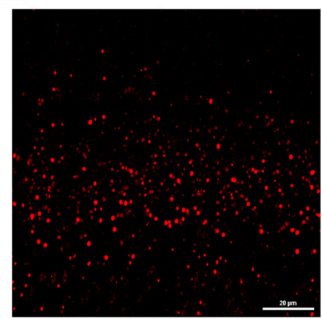

anti-beta galactosidase

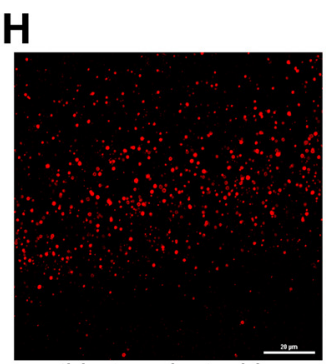

anti-beta galactosidase

K

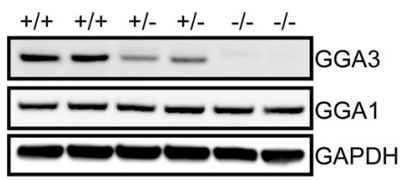

B

D

$\mathbf{F}$

$\mathbf{L}$
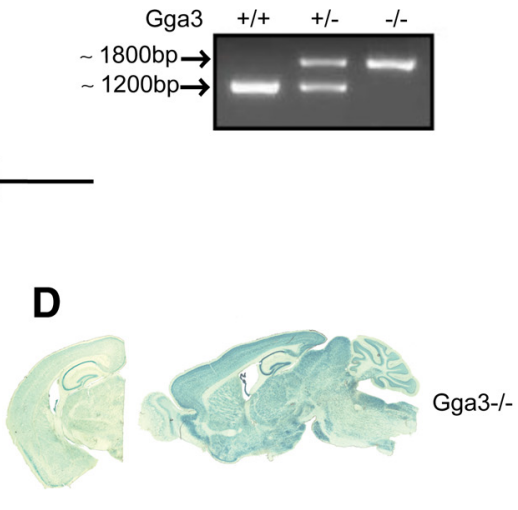

Gga3+1+

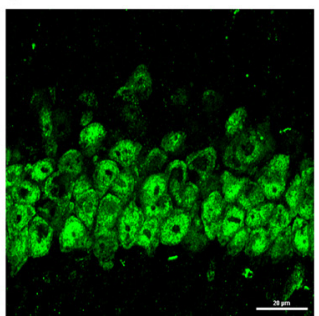

anti-NeuN

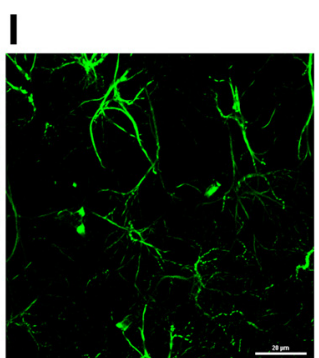

anti-GFAP

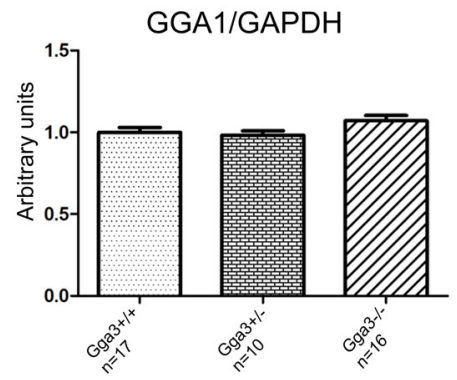

Figure 2. GGA3-null mice are healthy, viable, and fertile at 6 months of age. $A$, Schematic representation of the generation of the gene trapped Gga3 allele on mouse chromosome 11 using gene trap vector (BayGenomics). Gene trap vector inserted at nt 1173 of intron 1. $B, P C R$ genotyping of three offspring from the mating of two heterozygous mice using the three PCR primers indicated in $\boldsymbol{A}$. $\boldsymbol{C}$, Cresyl violet stained $30 \mu \mathrm{m}$ coronal sections from 6 -month-old $G g a 3^{-/-}$and $G g a 3^{+/+}$mice. $\boldsymbol{D}, \beta$-Galactosidase enzymatic staining of $30 \mu \mathrm{m}$ coronal and $60 \mu \mathrm{m}$ longitudinal frozen brain sections from 6-month-old Gga3 $3^{-/-}$and $\mathrm{Gga3^{+/+ }}$ mice. $\boldsymbol{E}-\boldsymbol{G}$, Confocal images of the CA1 region of the hippocampus from a $\mathrm{Gga3^{-/ }}$ mouse brain at $60 \times$ magnification (oil immersion) costained with antibodies against $\beta$-galactosidase ( $\boldsymbol{E}$, red; 559761, MP Biomedicals); NeuN ( $\boldsymbol{F}$, green; MAB377, Millipore), and overlayed image (G). Scale bar, $20 \mu \mathrm{m}$. $\boldsymbol{H}-\boldsymbol{J}$, Confocal images of the CA1 region of the hippocampus from a

2008). BACE1 haploinsufficiency results in a minimal reduction of $\mathrm{A} \beta$ in $\mathrm{BACE} 1\left(^{+/-}\right) /$ APP transgenic mice at 3 months of age and a $\sim 90 \%$ and $50 \%$ reduction of $A \beta$ levels at 13 and 18 months of age, respectively (McConlogue et al., 2007). More importantly, $\beta$-secretase activity has been shown to increase with age in human, monkey, and mouse brain (Fukumoto et al., 2004). Given that the impact of BACE1 levels on $\mathrm{A} \beta$ production seems to be age-dependent, the deletion of GGA3 may not result in increased $\mathrm{A} \beta$ production in young adult mice explaining the discrepancies we observed in vitro and in vivo.

\section{BACE1 levels and activity are increased in the Hippocampi of aged GGA3-null mice}

To assess the effect of GGA3 deletion on BACE1 elevation, $\beta$-secretase activity, and $\mathrm{A} \beta$ production during aging, we analyzed the hippocampi from 6-month-old and 18-24-month-old $\mathrm{Gga3}^{+/+}$and $\mathrm{Gga3}^{-/-}$ mice of both sexes. We chose to analyze the hippocampi of mice rather than their hemibrains as both GGA3 and BACE1 are highly expressed in this brain region. We found that, in agreement with our previous data in hemibrain extracts (Fig. $3 D$ ), BACE1 levels were increased by $25 \%$ ( $p=$ 0.0001) in the hippocampi of $\mathrm{Gga}^{-/-}$ mice at 6 months of age compared with their WT littermate controls. This increase in BACE1 was replicated in the hippocampi of aged $\mathrm{Gga3}^{-/-}$mice when compared with their WT littermate controls $(27 \% p<0.0001)$ (Fig. $4 A, B)$. Interestingly, overall levels of BACE1 did not increase in the hippocampi of $\mathrm{Gga}^{+/+}$ and $\mathrm{Gga3}^{-/-}$mice with aging but rather decreased in both $\mathrm{Gga3}^{+/+}$and $\mathrm{Gga3}^{-/-}$ aged mice when compared with their 6-month-old genetic counterparts $(\sim-17$ $p<0.0001$ and $-18 \%$, respectively, $p=$ $0.0011)$. However, the percentage elevation between genotypes remained the same (Fig. $4 B)$. In contrast, we did not detect any difference in PS1 levels (as a measure of $\gamma$-secretase) between $\mathrm{Gga3}^{-/-}$and their WT littermate controls at either 6 months or

\section{$\leftarrow$}

$\mathrm{Gga}^{-/-}$mouse brain at $60 \times$ magnification (oil immersion) costained with antibodies against $\beta$-galactosidase $(\boldsymbol{H}$, red; 559761, MP Biomedicals); GFAP (I, green; GA5, Millipore), and overlayed image (J). Scale bar, $20 \mu \mathrm{m}$. $\boldsymbol{K}$, Western blot analysis of the GGA3 (4167, Cell Signaling Technology) and GGA1 (H-215, Santa Cruz Biotechnology) levels in 6-month-old mouse brain lysates. $L$, The graph represents GGA1 levels normalized to GAPDH (mean \pm SEM) of $17 \mathrm{Gga3}^{+/+}, 10$ $\mathrm{Gga3}^{+/-}$, and $16 \mathrm{Gga}^{-/-}$mice and expressed as arbitrary units. 
A

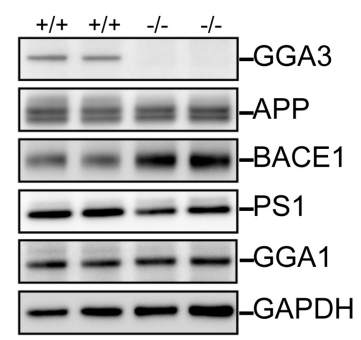

B

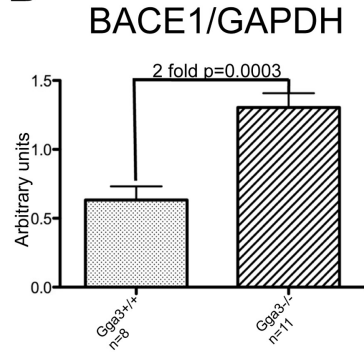

E

D
C

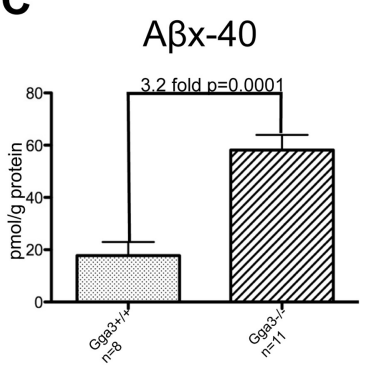

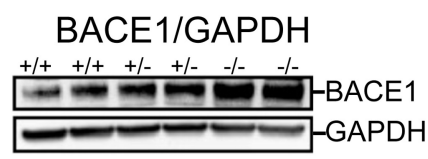

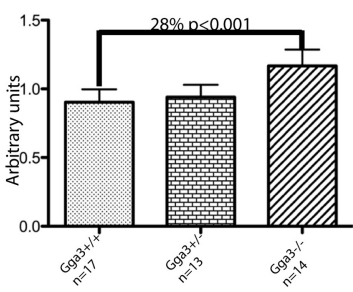

$\mathbf{F}$
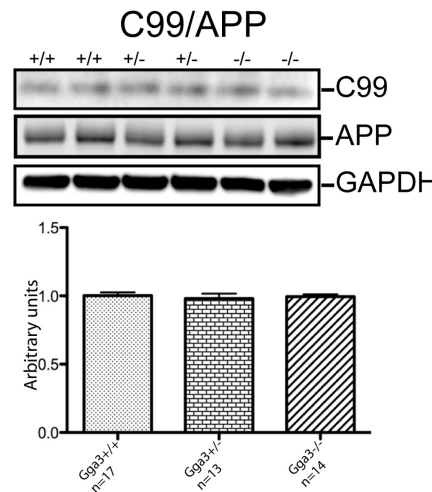

H

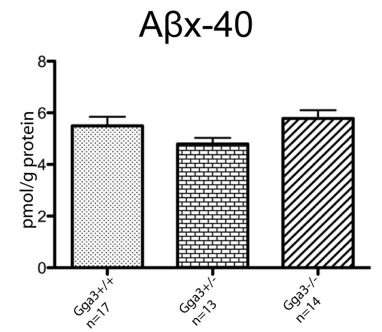

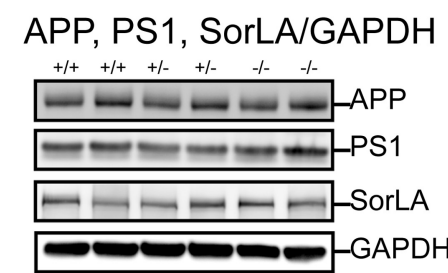

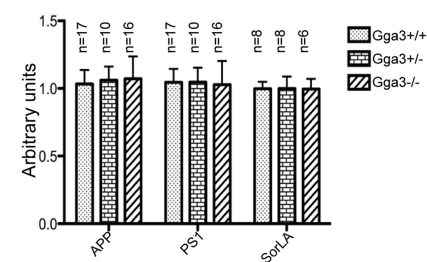

G
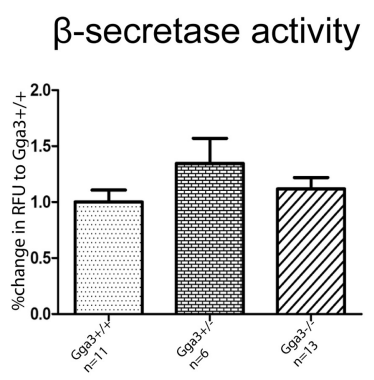

Figure 3. Genetic deletion of GGA3 increases levels of BACE1 in vitro and in vivo. $\boldsymbol{A}$, Western blot analysis of cell lysates from DIV 8 primary neuron cultures from $\mathrm{Gga} 3^{-/-}$and $\mathrm{Gga} 3^{+/+} \mathrm{P} 1$ mice. GGA3 (4167, Cell Signaling Technology), APP (m3.2), BACE1 (PA1-757, Thermo Scientific), PS1 (Ab14), GGA1 (H-215, Santa Cruz Biotechnology ). B, BACE1 levels were normalized to GAPDH and were increased twofold ( $p=0.0003)$. C, Secreted $A \beta 40$ was measured in conditioned media using the WAKOII Human/rodent $\mathrm{A} \beta \mathrm{x}-40$ ELISA. $\mathrm{A} \beta \mathrm{x}-40$ was increased 3.2-fold $(p=0.0001)$ in media from $\mathrm{Gga} 3^{-/-}$neurons. $\boldsymbol{D}$, Western blot analysis of BACE1 levels in brain lysates from 6-month-old mice using BACE1 antibody (PA1-757, Thermo Scientific). BACE1 levels normalized to GAPDH and represented as arbitrary units. BACE1 levels are elevated $28 \%(p<0.001)$ in $\mathrm{Gga}^{-/-}$mice compared with $\mathrm{Gga3^{+/+ }}$ littermates. $\boldsymbol{E}$, Western blot analysis of APP-full length (fl) (m3.2, Dr. Paul Mathews), presenilin-1 (Ab14, Dr. S. Gandy), SorLA (G25020, BD Transduction) levels in 6-month-old $\mathrm{Gga}^{+/+}, \mathrm{Gga}^{+/-}$, and $\mathrm{Gga}^{-/-}$mouse brain lysates. APP-fl, PS1, and SorLA normalized to GAPDH and represented as arbitrary units. No difference was observed in the levels of the proteins across the genotypes indicating that the GGA3 deletion is specific for BACE1.F, C99 levels were measured by Western blot analysis using m3.2 antibody in brain lysates. $(99$ levels normalized to APP-fl and represented as arbitrary units. No difference in C 99 levels was observed between genotypes. $\mathbf{G}, \beta$-Secretase activity assay measured by cleavage of the DABCYL/EDANS-conjugated

18-24 months of age (data not shown). Analysis of APP levels revealed that, in agreement with our previous data in hemibrain extracts (Fig. 3E), there was no difference in APP levels in the hippocampi of 6-month-old $\mathrm{Gga3}^{+/+}$and $\mathrm{Gga3}^{-/-}$mice. Instead, there was a significant decrease $(13 \%, p=0.0061)$ in APP levels in aged $\mathrm{Gga3}^{-\prime-}$ mice compared with their WT littermate controls (Fig. 4A,C). As was observed with BACE1 levels over aging, APP levels declined overall with aging in both $\mathrm{Gga3}^{+/+}$and $\mathrm{Gga3}^{-/-}$mice, $-21 \%(p<$ $0.0001)$ and $-31 \%(p<0.0001)$, respectively (Fig. 4C). When we analyzed levels of $\beta$-CTF (C99) normalized to full-length (fl) APP, we did not detect a difference in C99 levels between $\mathrm{Gga3}^{-/-}$mice and their WT littermate controls at 6 months of age in agreement with our previous data (Fig. $3 F$ ). However, C99 levels were increased by $21 \%$ $(p=0.0043)$ in aged $\mathrm{Gga3}^{-1-}$ mice when compared with their WT littermate controls (Fig. $4 A, D$ ). The increase in $\beta$-secretase activity in aged $\mathrm{Gga3}^{-1-}$ mice compared with their WT littermate controls as observed by an increase in C99 levels was confirmed by using a sensitive FRET-based $\beta$-secretase cleavage assay, which showed that $\beta$ secretase activity was increased by $70 \%$ ( $p=0.0128)$ in the hippocampi of aged $\mathrm{Gga3}^{-1-}$ mice compared with WT littermate controls (Fig. 4E). However, despite increased $\beta$-secretase levels and activity in the hippocampi of aged $\mathrm{Gga}^{-/-}$mice, we did not detect increased $A \beta$ levels in the hippocampus of the aged $\mathrm{Gga3}^{-/-}$mice compared with their WT controls (Fig. $4 F)$. Similar results were also obtained in cortical extracts from both 6-month-old and 18-24-month-old mice (data not shown). The significant decrease in APP along with an increase in C99 levels observed in aged $\mathrm{Gga}^{-/-}$mice is most likely the result of increased $\beta$-secretase activity. Accordingly, previous reports have shown

\section{$\leftarrow$}

$\beta$-secretase substrate IV measured in 6-month-old $\mathrm{Gga}^{+/+}$, $\mathrm{Gga}^{+/-}$, and $\mathrm{Gga}^{-/-}$mouse brain lysates. Data are represented as percentage change in Relative Fluorescence Units (RFU) of $\mathrm{Gga3}^{-/-}$and $\mathrm{Gga3}^{+/-}$when normalized to $\mathrm{Gga}^{+/+}$littermate controls. No statistically significant difference was observed in $\beta$-secretase activity between genotypes. $\boldsymbol{H}, A \beta x-40$ levels were analyzed in mouse brain lysates using the WAKOII Human/rodent $A \beta x-40$ ELISA. No difference was observed in $A \beta x-40$ across the genotypes. The data indicate mean \pm SEM of $17 \mathrm{Gga3}^{+/+}, 10 \mathrm{Gga3}^{+/-}$, and 16 $\mathrm{Gga}^{-/-}$for BACE1 and C99 levels; $11 \mathrm{Gga}^{+/+}, 6 \mathrm{Gga}^{+/-}$, and $13 \mathrm{Gga3}^{-/-}$mice for $\beta$-secretase activity; and of 18 $\mathrm{Gga3}^{+/+}, 13 \mathrm{Gga}^{+/-}$, and $14 \mathrm{Gga3}^{-/-}$mice for $\mathrm{A} \beta \mathrm{Xx}-40$. Statistical analysis of densitometry data was performed using an unpaired $t$ test with Welch correction. 
A

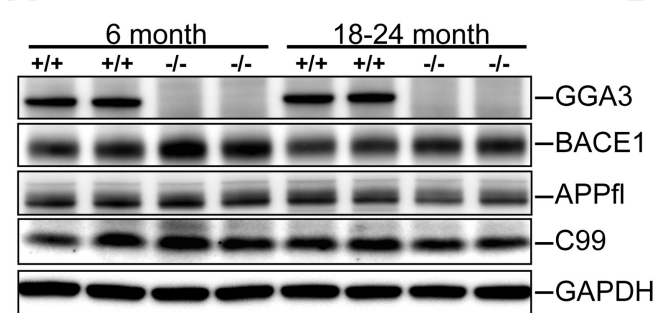

B

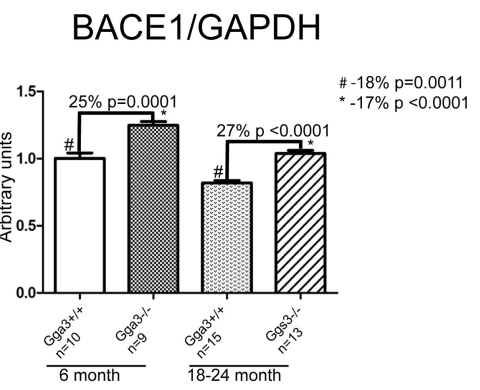

C

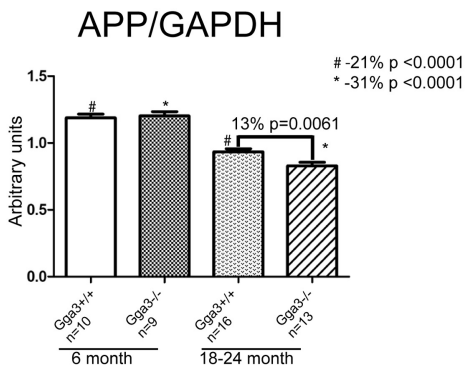

E

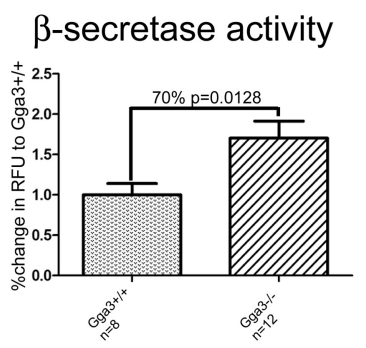

D

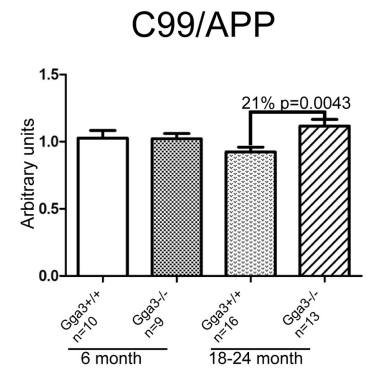

$\mathbf{F}$

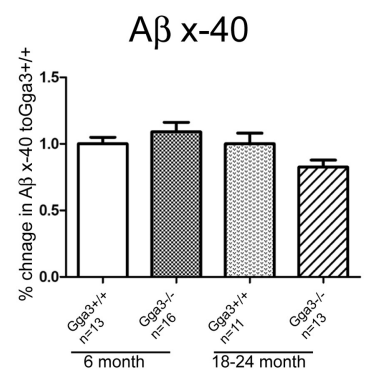

Figure 4. BACE1 levels and activity are increased in the Hippocampi of aged Gga $3^{-1-}$ mice. $A$, Western blot analysis of hippocampal lysates from 6-month-old and 18 -24-month-old $\mathrm{Gga}^{-/-}$and $\mathrm{Gga3^{+/+ }}$ mice. GGA3 (4167, Cell Signaling Technology), APP (m3.2), BACE1 (D10E5, Cell Signaling Technology), 99 (m3.2), and GAPDH (MAB374, Millipore). $\boldsymbol{B}$, The graph represents BACE1 levels normalized to GAPDH and reported as arbitrary units. BACE1 levels are elevated $25 \%(p=0.001)$ and $27 \%$ $(p<0.0001)$ in $\mathrm{Gga}^{-/-}$mice compared with $\mathrm{Gga}^{+/+}$littermates at 6 months of age and $18-24$ months of age, respectively. \#BACE1 levels are reduced $18 \%(p=0.0011)$ in the hippocampus of $\mathrm{Gga} 3^{+/+}$mice over aging. ${ }^{*} \mathrm{BACE} 1$ levels are reduced $17 \%$ $(p<0.0001)$ in the hippocampus of $\mathrm{Gga}^{-/-}$mice over aging. $C$, The graph represents APP levels normalized to GAPDH and reported as arbitrary units. APP-fl levels are reduced $13 \%(p=0.0061)$ in the hippocampus of $\mathrm{Gga}^{-1-}$ mice compared with

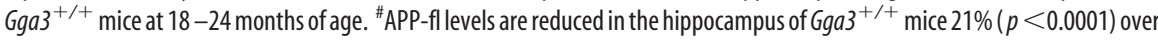
aging. *APP-fl levels are reduced in the hippocampus of $\mathrm{Gga}^{-1-}$ mice $31 \%(p<0.0001)$ over aging. $\boldsymbol{D}$, The graph represents $C 99$ levels normalized to APP-fl and reported as arbitrary units. C99 levels are increased 21\% ( $p=0.0043$ ) in the hippocampus of $\mathrm{Gga3}^{-/-}$mice compared with their $\mathrm{Gga3}^{+/+}$littermate controls at $18-24$ months of age. $\boldsymbol{E}, \beta$-Secretase activity assay measured by cleavage of the DABCYL/EDANS-conjugated $\beta$-secretase substrate IV measured in 18-24-month-old Gga3 ${ }^{+/+}$and $\mathrm{Gga}^{-/-}$mouse hemibrain lysates. Data are reported as percentage change in Relative Fluorescence Units (RFU) of $\mathrm{Gga} 3^{-1-}$ mice when normalized to $\mathrm{Gga} 3^{+/+}$littermate controls. $\beta$-Secretase activity is increased $70 \%(p=0.0128)$ in $\mathrm{Gga} 3^{-/-}$mice compared with $\mathrm{Gga3}^{+/+}$mice at $18-24$ months of age. $\boldsymbol{F}$, The graph represents $\mathrm{A} \beta \mathrm{x}-40$ levels in $\mathrm{Gga3^{+/+ }}$ and $\mathrm{Gga3^{-/- }}$ hippocampal lysates at 6 months and $18-24$ months of age. Data are reported as percentage change in $A \beta x-40$ when normalized to $\mathrm{Gga}^{+/+}$mice at each age. No difference was observed in $\mathrm{A} \beta \mathrm{x}-40$ between $\mathrm{Gga}^{+/+}$and $\mathrm{Gga}^{-/-}$mice at either age. The data indicate mean \pm SEM of $10 \mathrm{Gga}^{+/+}, 9 \mathrm{Gga}^{-/-}(6$ month old $), 16 \mathrm{Gga}^{+/+}$, and $13 \mathrm{Gga}^{-/-}(18-24$ month old $)$ mice for APP-fl and (99 levels; $10 \mathrm{Gga}^{+/+}, 9 \mathrm{Gga}^{-/-}\left(6 \mathrm{month}\right.$ old), $15 \mathrm{Gga}^{+/+}$, and $13 \mathrm{Gga}^{-/-}$(18-24 month old) mice for BACE1 levels; $8 \mathrm{Gga3}^{+/+}$and $12 \mathrm{Gga3}^{-/-} 18-24$-month-old mice for $\beta$-secretase activity; and $13 \mathrm{Gga}^{+/+}, 16 \mathrm{Gga3^{-/- }}(6$ month old), $11 \mathrm{Gga3}^{+/+}$, and $13 \mathrm{Gga}^{-/-}$(18-24 month old) mice for $\mathrm{A} \beta \mathrm{x}-40$. Statistical analysis of data was performed using an unpaired $t$ test with Welch correction.

that levels of murine full-length APP (APP-fl) are decreased while levels of $\beta$ APP-CTFs are increased in transgenic $(\mathrm{tg})$ mice expressing human (h) BACE1 (Bodendorf et al., 2002; Rockenstein et al., 2005; Lee et al., 2005). Our data demonstrating an increase in $\beta$-secretase activity without a further increase in BACE1 protein levels in the aged GGA3-null mice are in agreement with a previous study reporting that $\beta$-secretase activity increases without change in BACE1 protein levels in the mouse brain with aging (Fukumoto et al., 2004). Although GGA3 deletion leads to $\sim 30 \%$ increase in BACE1 levels and increased $\beta$-secretase activity with aging, such increase does not seem to be sufficient to exert a measurable effect on $A \beta$ levels.

\section{Head trauma potentiates BACE1} elevation induced by GGA3 deletion at $48 \mathrm{~h}$ after injury

To investigate the role of GGA3 on BACE1 elevation following experimental TBI, 6-month-old $\mathrm{Gga3}^{-/-}$, $\mathrm{Gga3}^{+/-}$, and $\mathrm{Gga3}^{+/+}$mice of both sexes were subjected to CCI. Western blot analysis of tissues collected $48 \mathrm{~h}$ after TBI revealed that BACE1 levels increase in the injured hemisphere of all mice (Fig. 5A). BACE1 levels were significantly increased $(13 \%, p=0.0359)$ in the injured hemisphere of $\mathrm{Gga3}^{-\prime-}$ mice compared with $\mathrm{Gga}^{+/+}$mice. The BACE1 levels were 30\% $(p=0.0011)$ higher in the contralateral hemisphere of $\mathrm{Gga3}^{-/-}$mice compared with the $\mathrm{Gga}^{+/+}$mice. This is comparable to the difference in BACE1 levels observed between $\mathrm{Gga3}^{-/-}$and $\mathrm{Gga3}^{+/+}$naive mice (i.e., mice not subjected to TBI, Fig. 3D). As a consequence of the elevated BACE1 levels in the $\mathrm{Gga}^{-/-}$contralateral hemisphere, the percentage increase in BACE1 levels between the injured and contralateral hemispheres in $\mathrm{Gga3}^{-/-}$mice was only $37 \%$ $(p=0.0031)$ compared with $60 \%(p<$ 0.0001) in $\mathrm{Gga3}^{+/+}$mice (Fig. $5 A$ ). Therefore, postinjury elevation of BACE1 is reduced by $\sim 50 \%$ in the GGA3-null mice. The percentage increase in BACE1 levels in the $\mathrm{Gga3}^{+/-}$mice $(33 \%, p=0.0020)$ was similar to that observed in the $G g a 3^{-/-}$ mice; however, GGA3 haploinsufficiency did not potentiate BACE1 elevation in the injured hemisphere but rather slightly increased BACE1 levels in the contralateral hemisphere (Fig. 5A). CCI produces a focal injury; however, modest alterations have been reported in the contralateral hemisphere depending on the severity of the injury (Hall et al., 2005), which may account for the increase observed in the contralateral hemisphere of $\mathrm{Gga3}^{+/-}$mice.

Next, we assessed $\beta$-secretase activity in brain extracts of mice subjected to CCI by measuring $\beta$-CTF (C99) levels, and found that $\mathrm{C} 99$ levels were significantly increased in the injured hemispheres of $\mathrm{Gga3}^{+/+}$and $\mathrm{Gga3}^{-1-}$ but not $\mathrm{Gga3}^{+/-}$mice. As was observed with BACE1 levels, the greatest percentage increase in $\beta$-secretase activity occurred in the $\mathrm{Gga3}^{+/+}$mice $(92 \%, p=0.0017)$ compared with $\mathrm{Gga3}^{-/-}$mice $(70 \%, p<0.0001$ ) (Fig. 5B). APP C99 levels were significantly 
increased $(25 \%, p=0.0172)$ in the contralateral hemisphere of $\mathrm{Gga3}^{-/-}$mice compared with $\mathrm{Gga3}^{+/+}$mice, accounting for the smaller percentage increase observed (Fig. 5B). The increase in $\beta$-secretase activity observed in the contralateral hemisphere of $\mathrm{Gga3}^{-1-}$ mice compared with $\mathrm{Gga}^{+/+}$mice after TBI is interesting as we did not observe an increase in naive mice (i.e., not subjected to TBI), and it is most likely due to the modest global effects that have been noted by other researchers following CCI (Hall et al., 2005). A $\beta 40$ levels were significantly increased in the injured compared with contralateral hemispheres of $\mathrm{Gga}^{+/+}$and $\mathrm{Gga3}^{-/-}$but not $\mathrm{Ggal}^{+/-}$mice similarly to APP C99 levels. (Fig. 5C). Levels of PS1 and APP were unchanged between the injured and contralateral hemisphere across genotypes (data not shown).

While TBI potentiates BACE1 elevation in GGA3-null mice, $A \beta$ levels were similar in the injured hemisphere of $\mathrm{Gga3}^{+/+}$and $\mathrm{Gga3}^{-/-}$mice. One possible explanation is that following TBI, levels of $\mathrm{A} \beta$ degrading enzymes (e.g., neprilysin) are upregulated (Chen et al., 2009), which may account for the observed dissociation between BACE1 and $A \beta$ levels. Alternatively, it has been reported that high expression levels of BACE1 suppress $\mathrm{A} \beta$ production both in vitro and in vivo (Creemers et al., 2001; Lee et al., 2005). Thus, it is possible that the robust accumulation of BACE1 in GGA3-null mice following TBI produces a similar suppression of $\mathrm{A} \beta$ production.

Analysis of cavitary lesion volume in a subset of the $\mathrm{Gga3}^{+/+}, \mathrm{Gga3}^{+/-}$, and $\mathrm{Gga3}^{-/-}$mice demonstrated that while GGA3 depletion potentiates BACE1 elevation in the acute phase following TBI, it is insufficient to cause a measurable increase in lesion volume 2 weeks after injury (mean \pm SEM of 7 mice per genotype: $\mathrm{Gga3}^{+/+}$: $7.25 \pm 0.32 \mathrm{~mm}^{3} ; \mathrm{Gga3}^{+/-}: 7.22 \pm 0.19 \mathrm{~mm}^{3} ; \mathrm{Gga3}^{-/-}$: $\left.7.57 \pm 0.29 \mathrm{~mm}^{3}\right)$.

In summary, we determined that TBI potentiates BACE1 elevation in $\mathrm{Gga3}^{-/-}$mice at $48 \mathrm{~h}$ after injury. Consequently, these findings indicate that in addition to the GGA3-mediated posttranslational stabilization of BACE1, other mechanisms also contribute to BACE1 accumulation in the acute phase after injury.

\section{GGA1 is depleted in the acute phase after TBI}

In an effort to find additional mechanisms responsible for the BACE1 elevation observed $48 \mathrm{~h}$ after TBI in the $\mathrm{Gga3}^{-1-}$ mice and given that previous reports have shown that GGA1 levels are significantly decreased $(\sim 40 \%)$ in $\mathrm{AD}$ brains (Wahle et al., 2006), we set out to determine whether GGA1 is depleted concurrently with GGA3 following TBI. We found that GGA3 levels were decreased in the injured versus contralateral hemisphere of $\mathrm{Gga3}^{+/+}$and $\mathrm{Gga3}^{+/-}$mice $(55 \% \mathrm{p}=0.0002$ and $48 \%$
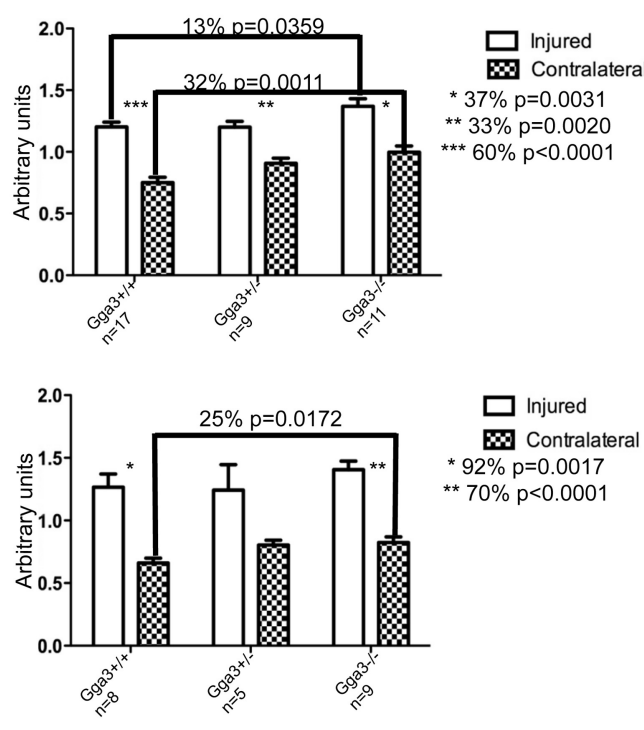
GAPDH

Figure 5. Head trauma potentiates BACE1 elevation induced by GGA3 deletion at $48 \mathrm{~h}$ after injury. $A$, Western blot analysis of BACE1 levels in brain lysates from 6-month-old mice $48 \mathrm{~h}$ after TBI using BACE antibody (PA1-757, Thermo Scientific). The graph

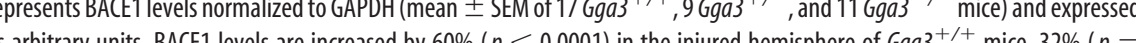
$0.0020)$ in the injured hemisphere of $\mathrm{Gga}^{+/-}$mice, and $37 \%(p=0.0031)$ in the injured hemisphere of $\mathrm{Gga}^{-/-}$mice. $\boldsymbol{B}$,

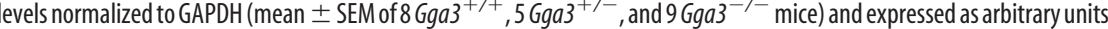

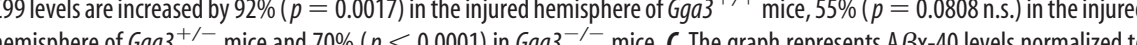
hemisphere of $\mathrm{Gga3}^{+/+}$mice, 20\% ( $p=0.1650$ n.s.) in the injured hemisphere of $\mathrm{Gga3}^{+/-}$, and $18 \%(p=0.0052)$ in the injured hemisphere of $\mathrm{Gga}^{-1-}$ mice. Statistical analysis of data was performed using a paired $t$ test.

$p=0.0184$, respectively) (Fig. 6A,B). Levels of GGA1 were also decreased in the injured hemispheres of $\mathrm{Gga3}^{-1-}(61 \%, p<$ $0.0001), \mathrm{Gga3}^{+/-}(53 \%, p=0.0019)$, and $\mathrm{Gga3}^{+/+}(67 \%, p<$ 0.0001 ) mice $48 \mathrm{~h}$ after injury (Fig. 6A,C). However, unlike GGA3 there was no difference in residual GGA1 levels observed in the injured hemispheres across genotypes.

\section{Caspase- 3 cleaves GGA1 at D306 generating a dominant-negative molecule}

Caspase activation is a well known mechanism of programmed cell death following TBI in both humans and experimental models (Clark et al., 1999, 2000; Knoblach et al., 2002; Chen et al., 2004). To determine whether GGA1 is depleted during caspase activation H4-APP751 cells were treated with STS alone or in association with a general caspase inhibitor (zVAD) for $16 \mathrm{~h}$. Western blot analysis with an anti-GGA1 antibody, targeted to the hinge and GAE domains of GGA1 (amino acids 286-500), revealed that full-length GGA1 is cleaved into several fragments 


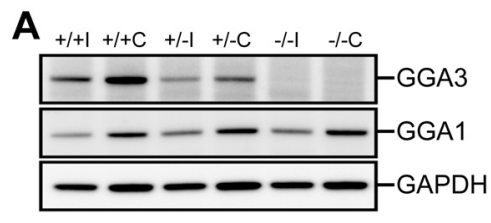

B GGA3/GAPDH

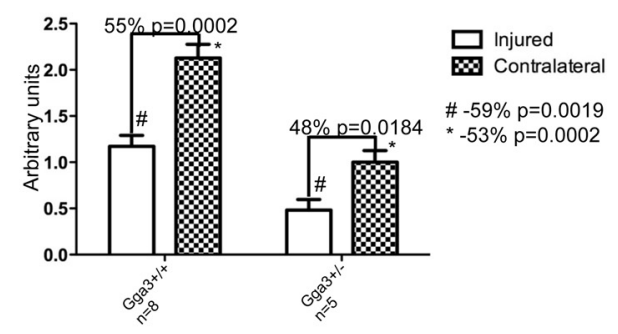

C

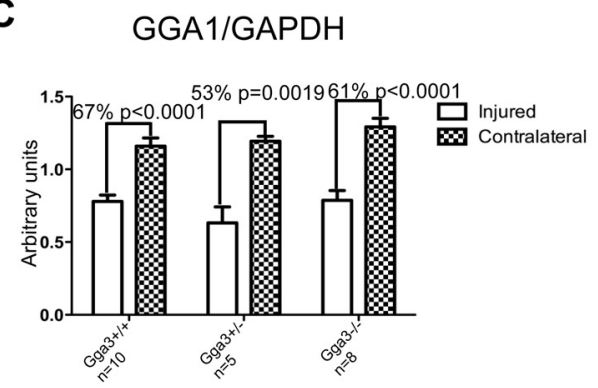

Figure 6. GGA1 is depleted in the acute phase after TBI. $A$, Western blot analysis of GGA 3 and GGA1 levels in $\mathrm{Gga}^{+/+}, \mathrm{Gga}^{+/-}$, and $\mathrm{Gga}^{-/-}$mice $48 \mathrm{~h}$ after injury. B, The graph represents GGA3 levels normalized to GAPDH (mean \pm SEM of $8 \mathrm{Gga}^{+/+}, 5 \mathrm{Gga}^{+/-}$) and expressed as arbitrary units. GGA3 levels are reduced by $55 \%(p=0.002)$ in the injured hemisphere of $\mathrm{Gga}^{+/+}$mice and $48 \%(p=0.0184)$ in the injured hemisphere of $\mathrm{Gga}^{+/-}$ mice at 48 h after injury. ${ }^{\# G G A 3}$ levels are reduced $59 \%(p=0.0019)$ in the injured hemisphere of $\mathrm{Gga}^{+/-}$mice compared with $\mathrm{Gga}^{+/+}$mice. ${ }^{*} \mathrm{GGA}$ levels are reduced 53\% ( $\left.p=0.0002\right)$ in the contralateral hemisphere of $\mathrm{Gga} 3^{+/-}$mice compared with $\mathrm{Gga}^{+/+}$mice. C, The graph represents GGA1 levels normalized to GAPDH (mean \pm SEM of $10 \mathrm{Gga}^{+/+}, 5 \mathrm{Gga}^{+/-}$and 8 $\left.\mathrm{GGA3}^{-1-}\right)$ and expressed as arbitrary units. GGA1 levels are reduced by $67 \%(p<0.001)$ in the injured hemisphere of $\mathrm{GGA3}^{+/+}$mice, by $53 \%(p=0.0019)$ in the injured hemisphere of $\mathrm{GGA3}^{+/-}$mice, and by $61 \%(p<0.0001)$ in the injured hemisphere of $\mathrm{Gga}^{-1-}$ mice $48 \mathrm{~h}$ after injury. Statistical analysis of data was performed using a paired $t$ test.

during apoptosis and that caspase inhibition (zVAD treatment) prevents GGA1 depletion (Fig. 7A).

To determine whether GGA1 is a caspase-3 substrate, we subjected in vitro translated (IVT) GGA3 and GGA1 to incubation with increasing concentrations of recombinant caspase-3 overnight at $37^{\circ} \mathrm{C}$. Western blot analysis of the IVT extracts using antibodies specific for GGA3 and GGA1 demonstrate that GGA1 is capable of being cleaved by caspase- 3 and its cleavage generates a fragment pattern similar to that of GGA3 (Fig. 7B). We have previously shown that caspase-3 cleaves GGA3 at D313 and generates a dominant-negative molecule (Tesco et al., 2007); therefore, we assessed whether GGA1 is cleaved at the corresponding aspartic acid residue (a.a. 306) (Fig. 7C). We mutagenized the D306 residue in the wild-type myc-tagged GGA1 plasmid to an alanine and subjected both wild-type Myctagged GGA1 (w.t.) and mutated GGA1 (D306A) to in vitro translation followed by caspase-3 cleavage. Western blot analysis with an anti-Myc antibody revealed that the D306A mutation prevented the generation of a specific caspase-3-derived fragment (Fig. $7 D$, Fragment 1 D.N.). The GGA1-truncated molecule ending at D306 contains the VHS and GAT domain, which has been shown to function as a dominant-negative by attenuating the retrograde transport of BACE1 from endosomes to the TGN (Wahle et al., 2005; Wahle et al., 2006). Thus, during apoptosis, caspase-mediated cleavage of GGA1 results not only in the degradation of GGAl, but also in the production of a GGA1 dominant-negative molecule.

\section{RNAi silencing of GGA1 potentiates BACE1 elevation induced by GGA3 deletion}

To investigate whether GGA1 depletion potentiates the BACE1 elevation induced by GGA3 deletion, GGA1 was silenced in primary cortical neurons (PCN) collected from P1 $\mathrm{Gga}^{-/-}$pups using a lentivirus encoding a GGA1 shRNA. PCNs were infected with either a lentivirus encoding a GGA1shRNA or negative control shRNA at a MOI of 5 for $6 \mathrm{~h}$ on DIV3. Cells were collected at DIV15 and analyzed by Western blotting (Fig. $8 A$ ). BACE1 levels were increased $60 \%(p=0.0029)$ in $\mathrm{Gga3}^{-/}$cortical neurons depleted of GGAl compared with those expressing a negative control shRNA (Fig. 8A). While levels of PS1 were unchanged, APP levels (in particular the immature isoform of APP) were significantly increased in cortical neurons depleted of GGA1 (26\% increase $p=0.0179$ ) (Fig. 8A). This increase in APP is specifically due to the depletion of GGA1 as we have previously shown that GGA3 depletion does not affect APP levels either in vitro or in vivo (Tesco et al., 2007) (Fig. 3A). The observed increases in BACE1 and APP levels appear to be specific for the deletion of GGA3 and GGA1 as caspase activation was ruled out by the detection of unchanged levels of full-length caspase-3 (Fig. $8 A$ ). These data indicate that GGA3 and GGA1 synergistically regulate BACE1 degradation and that caspase-mediated depletion of GGAl is a leading candidate mechanism to explain BACE1 elevation in $\mathrm{Gga3}^{-/-}$mice at $48 \mathrm{~h}$ after injury.

\section{GGA3 and GGA1 but not GGA2 are depleted in AD brains}

Several studies have demonstrated that BACE1 levels and activity are elevated in the brains of AD patients (Fukumoto et al., 2002; Holsinger et al., 2002; Tyler et al., 2002; Yang et al., 2003; Li et al., 2004). We have previously shown that GGA3 levels are significantly decreased (55\%) and inversely correlated with BACE1 levels in the temporal cortex of patients with $\mathrm{AD}$ (Tesco et al., 2007). We reanalyzed this cohort of patients for GGA1 and GGA2 levels and demonstrated that like GGA3, GGA1 levels are also decreased in the temporal cortex of AD sufferers ( $30 \%$ decrease, $p=0.02$; Fig. $8 B, C$ ), while levels of GGA2 levels are unchanged. Together, these data suggest that depletion of both GGA3 and GGA1 contributes to the BACE1 elevation observed in $\mathrm{AD}$ brains.

\section{GGA3 haploinsufficiency results in sustained elevation of BACE1 and $A \beta$ levels in the subacute phase of injury} We have previously demonstrated that GGA3 regulates BACE1 degradation by trafficking BACE1 to the lysosomes (Tesco et al., 2007; Kang et al., 2010). Thus, GGA3 is expected to play a key role in the disposal of the BACE1 accumulated during the acute phase after injury. Given that GGA3 levels are decreased by $\sim 50 \%$ in the temporal cortex of AD patients, $\mathrm{Gga3}^{+/-}$mice best represent the GGA3 reduction observed in the AD brains. In an attempt to address the important question of how acute brain injuries (e.g., stroke and head trauma) result in chronic accumulation of $A \beta$, we investigated the effect of GGA3 haploinsufficiency on BACE1 levels in the subacute phase of injury ( $7 \mathrm{~d}$ after TBI). Six-monthold $\mathrm{Gga}^{+/-}$and $\mathrm{Gga3}^{+/+}$mice of both sexes were subjected to CCI. Western blot analysis of tissues collected $7 \mathrm{~d}$ after TBI re- 
A
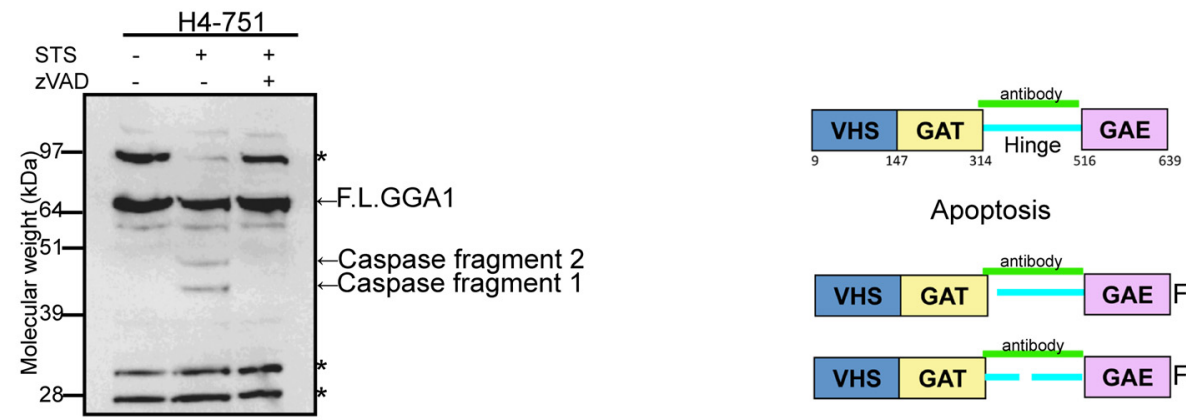

Apoptosis

\begin{tabular}{|l|l|l|}
\hline VHS & GAT & GAE \\
\hline VHS & GAT & Fragment 1 \\
\hline
\end{tabular}

B

GGA3 IVT

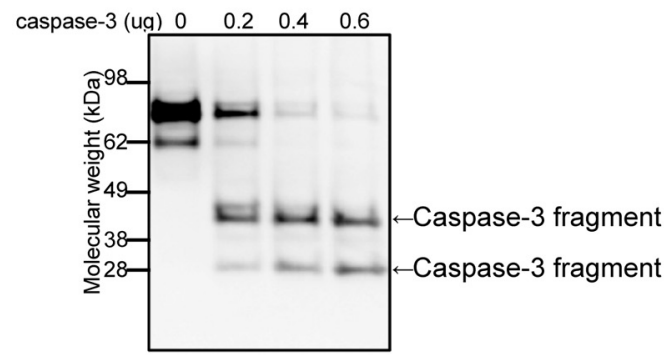

GGA1 IVT

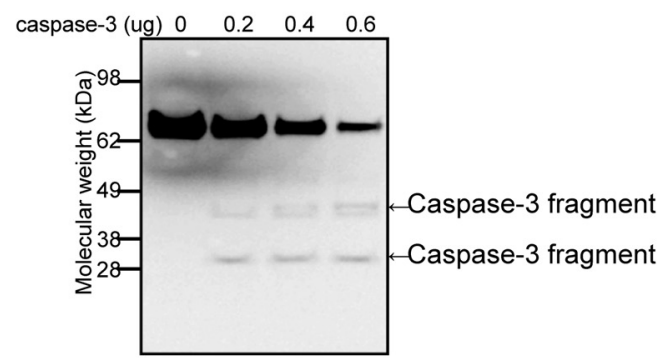

C

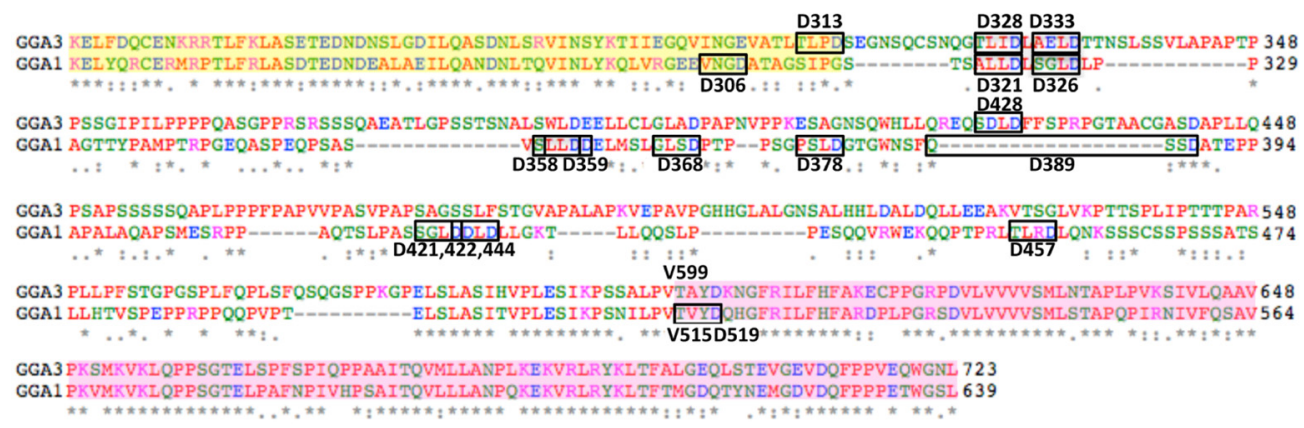

D

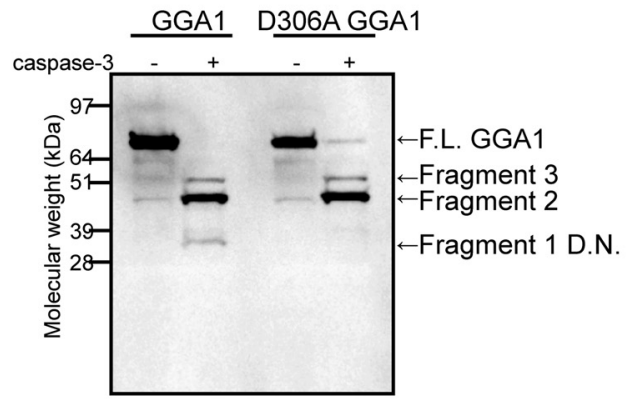

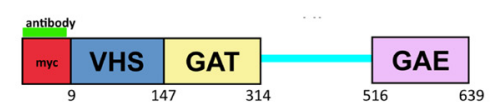

Apoptosis/Caspase-3

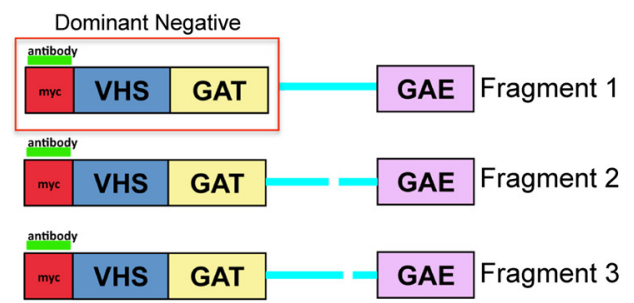

Figure 7. Caspase-3 cleaves GGA1 at D306 generating a dominant-negative molecule. $\boldsymbol{A}$, Western blot analysis of GGA1 cleavage (H-215, Santa Cruz Biotechnology) following STS-induced apoptosis in $\mathrm{H} 4-751$ cell extracts. $\mathrm{H} 4-751$ cells were incubated in the presence of $1 \mu \mathrm{m} \mathrm{STS}$ with or without $50 \mu \mathrm{m} \mathrm{ZVAD} \mathrm{at} 37^{\circ} \mathrm{C}$ for $16 \mathrm{~h}$. Cell lysates were electrophoresed on a $4-12 \%$ Bis-Tris acrylamide gel with MES running buffer. *Nonspecific bands not representative of apoptotic cleavage. The cleavage pattern detected with the H-215 antibody following apoptotic cleavage due to STS treatment is schematically represented. B, Western blot analysis of GGA3 caspase cleavage pattern using GGA3 antibody (4167, Cell Signaling Technology). In vitro translated HA-GGA3 subjected to $0 / \mathrm{N}$ recombinant caspase-3 cleavage at increasing amounts. Western blot analysis of GGA1 caspase cleavage pattern using GGA1 antibody (H-215, Santa Cruz Biotechnology). In vitro translated Myc-GGA1 subjected to 0/N recombinant caspase-3 cleavage at increasing amounts. Cell lysates were electrophoresed on a $4-12 \%$ Bis-Tris acrylamide gel with MES running buffer. Arrows denote caspase-3 cleavage fragments. C, Schematic representation of putative caspase-3 cleavage sites in the human GGA1 and GGA3 protein sequence. Aspartate 306 in GGA1 is the corresponding residue to Aspartate 313 in GGA3. D, Western blot analysis using an anti-Myc antibody (9B11, Cell Signaling Technology) of the recombinant caspase-3 cleavage of IVT Myc-GGA1 WT and Myc-GGA1D306A. Cell lysates were electrophoresed on a 4-12\% Bis-Tris acrylamide gel with MOPS running buffer. Caspase-3 cleavage of IVT GGA1 produces three caspase-derived fragments including a dominantnegative fragment as previously observed with GGA3. Mutation of Aspartate 306 to Alanine in GGA1 sequence prevents the formation of the dominant negative. Schematic representation of the GGA1 fragments produced by recombinant caspase-3 cleavage as detected by the anti-Myc antibody. 
A

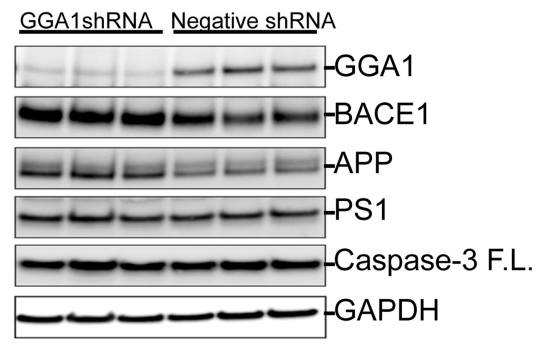

B

AD\#1 AD\#2 ND\#1 ND\#2 AD\#3 AD\#4 ND\#3 ND\#4

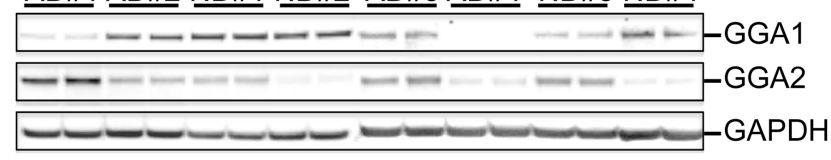

\section{GGA/GAPDH}

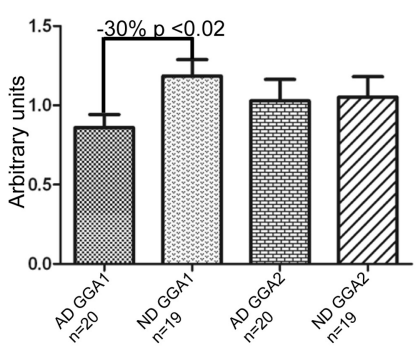

Figure 8. GGA3 and GGA1 are depleted in $A D$ brains and RNAi silencing of GGA1 potentiates BACE1 elevation induced by GGA3 deletion. $\boldsymbol{A}$, Western blot analysis of cell lysates from $\mathrm{Gga}^{-/-}$primary cortical neurons infected with lentivirus expressing either GGA1shRNA or negative control shRNA at an MO1 5. Cortical neurons were infected on DIV3 and collected DIV15. The following antibodies were used: BACE1 (D10E5, Cell Signaling Technology), APP-fl (m3.2), presenilin-1 (Ab14), GGA3 (4167, Cell Signaling Technology), and GGA1 (H-215, Santa Cruz Biotechnology). BACE1 levels were normalized to GAPDH and expressed as arbitrary units (mean \pm SEM of 7 replicates for GGA1shRNA and 5 replicates for negative control shRNA). BACE1 levels are increased by $60 \%(1.23 \pm 0.07$ vs $0.77 \pm 0.08, p=0.0029)$ in $\mathrm{Gga}^{-/-}$ neurons in which GGA1 is also depleted. APP levels were normalized to GAPDH and expressed as arbitrary units (mean \pm SEM of 7 replicates for GGA1shRNA and 5 replicates for negative control shRNA). APP (in particular the immature isoform) is increased $26 \%$ (1.14 \pm 0.07 vs $0.90 \pm$ $0.03, p=0.0179)$ in $\mathrm{Gga}^{-/-}$neurons depleted of GGA1. $\boldsymbol{B}$, Western blot analysis of the temporal cortex of human brains. GGA1 was detected using an anti-GGA1 antibody (gift from Margaret Robinson). GGA2 was detected using anti-GGA2 antibody (BD Transduction Laboratories). C, GGA1 and GGA2 densitometry values were normalized against GAPDH values. At least triplicates of each sample were analyzed. The graphs represent mean \pm SEM of 19 ND and 20 AD. Statistical analysis of data performed using an unpaired $t$ test with Welch correction.

vealed that BACE1 levels were similar in the injured and contralateral hemisphere of $\mathrm{Gga3}^{+/+}$mice. In contrast, BACE1 levels were still increased by $\sim 20 \%(p=0.0025)$ in the injured hemisphere of $\mathrm{Gga3}^{+/-}$compared with the contralateral hemisphere (Fig. 9A). Accordingly, C99 and A $\beta 40$ levels were increased by $\sim 40 \%(p=0.0313)$ and $\sim 25 \%(p=0.0075)$, respectively, in the injured versus contralateral hemisphere in $\mathrm{Gga3}^{+/-}$but not $\mathrm{Gga3}^{+/+}$mice (Fig. 9B,C). The observed increase in $\mathrm{A} \beta 40$ levels appears to be due to enhanced $\beta$-secretase activity, as APP and PS1 levels (a measure of $\gamma$-secretase) remain unchanged (data not shown). GGA1 levels were restored to normal while GGA3 was still slightly depleted in the injured hemisphere of both $\mathrm{Gga3}^{+/+}$ and $\mathrm{Gga}^{+/-}$mice at $7 \mathrm{~d}$ after injury (Fig. $9 D-F$ ). This demonstrates that while multiple mechanisms including depletion of GGA3 and GGA1 are responsible for the elevation of BACE1 in the acute phase after injury, in the subacute phase of injury haploinsufficiency of GGA3 is solely responsible for a sustained increase in BACE1 level and activity, and $\mathrm{A} \beta$ production.

\section{Discussion}

We report here a novel GGA1/3-mediated mechanism underlying BACE1 elevation following TBI. We have found that GGA3 is depleted while BACE1 levels increase in the acute phase after TBI. We have demonstrated the role of GGA3 in the regulation of BACE1 in vivo by showing that BACE1 levels are increased in the brain of GGA3-null mice and that GGA3 deletion leads to increased $\beta$-secretase activity with aging. We next asked to what extent the deletion of GGA3 affects BACE1 elevation following TBI; we found that head trauma potentiates BACE1 elevation in GGA3-null mice at $48 \mathrm{~h}$ after TBI. Consequently, these findings indicate that in addition to the GGA3-mediated posttranslational stabilization of BACE1, other mechanisms also contribute to BACE1 accumulation in the acute phase after injury.

In an effort to find other mechanisms responsible for the BACE1 elevation observed at $48 \mathrm{~h}$ after TBI, we discovered that GGA1 is depleted by caspase cleavage both in vitro following apoptosis and in vivo at $48 \mathrm{~h}$ after TBI. Furthermore, GGA1 silencing potentiates BACE1 elevation induced by GGA3 deletion in neurons in vitro. Thus, we conclude that depletion of GGA1 by RNAi-mediated silencing or by caspase activation following TBI potentiates the BACE1 elevation produced by GGA3 deletion in vitro and in vivo, respectively. Importantly, we have shown that decreased levels of GGA1 are associated with the depletion of GGA3 and BACE1 elevation observed in a series of postmortem $\mathrm{AD}$ brains. These findings confirm and extend a previous report showing that GGA1 levels were significantly decreased $(\sim 40 \%)$ in AD brains (Wahle et al., 2006). Collectively, our data indicate that depletion of GGA1 and GGA3 synergistically elevate BACE1 levels and suggest that the BACE1 elevation observed in $\mathrm{AD}$ brains is mediated by the concurrent depletion of GGAl and GGA3. Our data support a molecular mechanism by which BACE1 levels are regulated by caspase-mediated depletion of GGA1 and GGA3 in the acute phase of brain injury.

While caspase activation is a well known mechanism of programmed cell death following TBI in both humans and experimental models (Clark et al., 1999, 2000; Knoblach et al., 2002; Chen et al., 2004), the role of caspase activation in neurodegenerative diseases has been matter of debate for a very long time. In support of a role for caspase activation in $\mathrm{AD}$, we demonstrated that caspase- 3 is activated in the same series of AD brains analyzed here (Tesco et al., 2007). Moreover, recent reports have provided compelling evidence that caspase activation is an early event, which plays a key role in neurodegeneration. Using in vivo multiphoton microscopy in association with fluorescent dyes, de Calignon et al. (2010) demonstrated that caspase activation precedes tangle formation in Tau transgenic mice. Surprisingly, this same study showed that neurons, in which caspase activation occurs, do not die acutely, but develop tangles. Similarly, caspase- 3 activation is increased in hippocampal dendritic spines and is an early event associated with synaptic dysfunction in Tg2576 mice (D'Amelio et al., 2011). Caspase-3 activation also mediates the inhibition of LTP induced by $\mathrm{A} \beta 42$ toxicity (Frohlich et al., 2011). Thus, caspase-mediated depletion of GGA1 and GGA3 is a candidate mechanism contributing to BACE1 elevation in AD brains.

To date, several mechanisms have been proposed to explain the increased accumulation of BACE1 in AD brains: depletion of GGA3 (Tesco et al., 2007); increased phosphorylation of transla- 

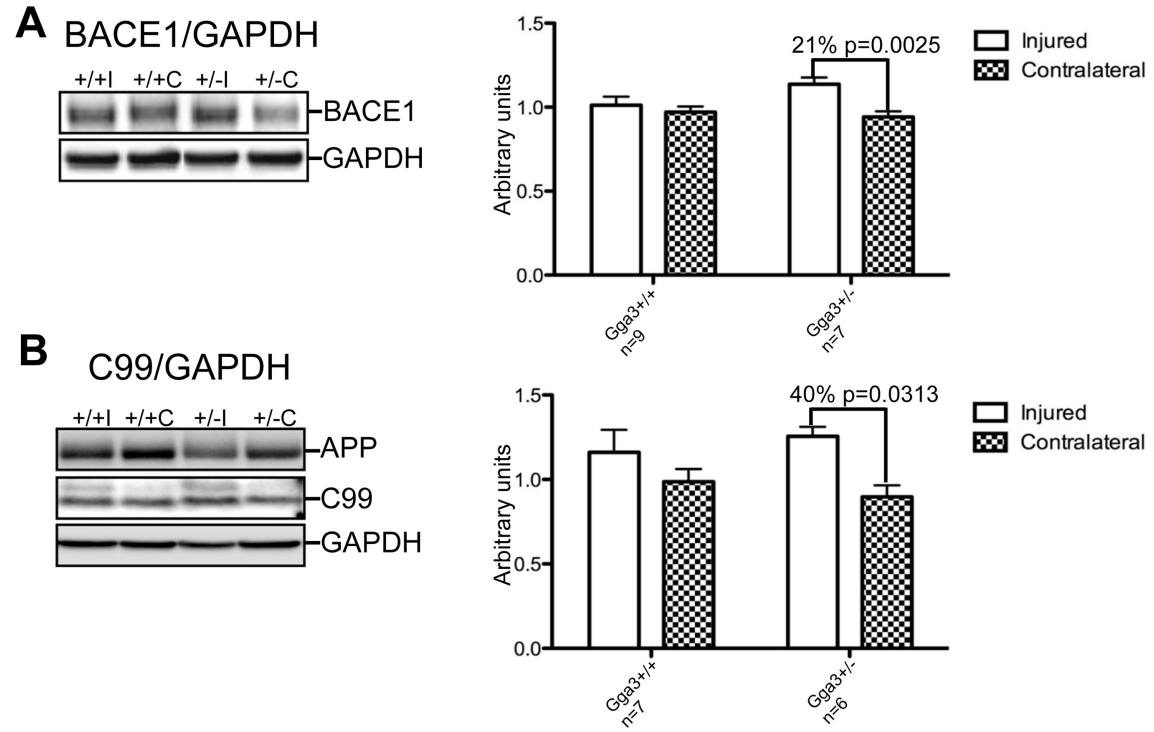

C

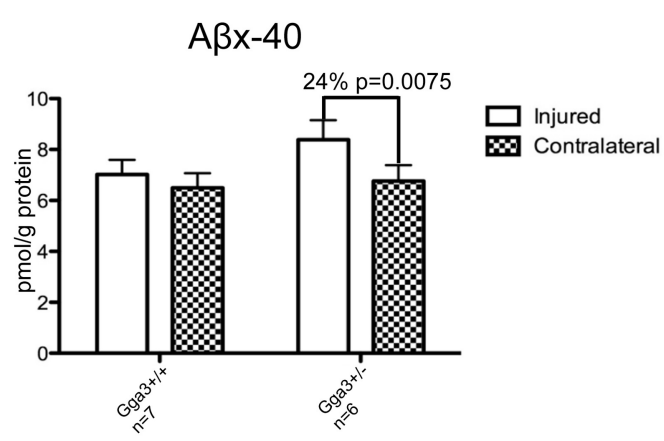

D

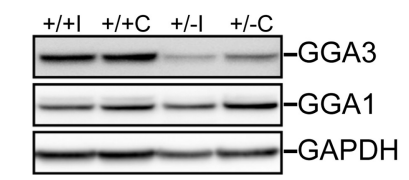

$\mathbf{F}$
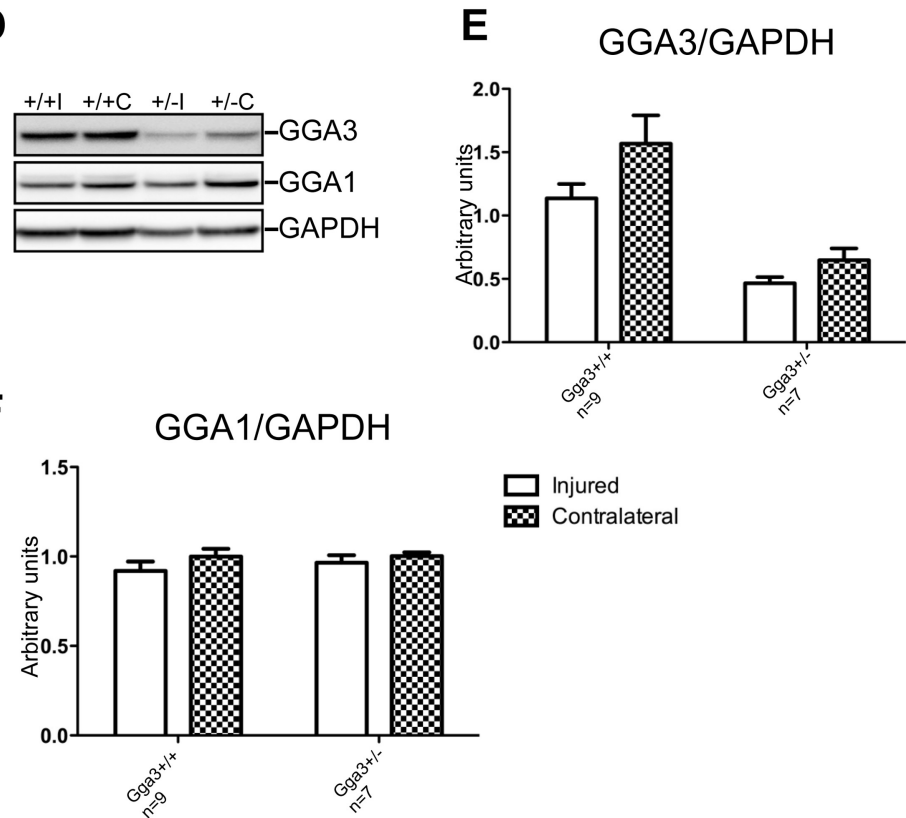

Figure 9. GGA3 haploinsufficiency results in sustained elevation of BACE1 and $A \beta$ levels in the subacute phase of injury. $\boldsymbol{A}$ Western blot analysis of BACE1 levels in brain lysates from 6-month-old mice $7 \mathrm{~d}$ after TBI using BACE1 antibody (PA1-757, Thermo Scientific). The graph represents BACE1 levels normalized to GAPDH (mean \pm SEM of $9 \mathrm{Gga3^{+/+ }}$ and $7 \mathrm{Gga3^{+/- }}$ mice) and expressed as arbitrary units. BACE1 levels are increased by $21 \%(p=0.0025)$ in the injured hemisphere of $\mathrm{Gga}^{+/-}$mice at $7 \mathrm{~d}$ after injury. $\boldsymbol{B}$, Western blot analysis of $\mathbf{C} 99$ levels in brain lysates from 6-month-old mice $7 \mathrm{~d}$ after TBI using m3.2 antibody. The graph represents $C 99$ levels normalized to GAPDH (mean \pm SEM of $7 \mathrm{Gga}^{+/+}$and $6 \mathrm{Gga3^{+/- }}$ mice) and expressed as arbitrary units. $C 9$ levels are increased by $40 \%(p=0.0313)$ in the injured hemisphere of Gga $3^{+/+}$mice $7 \mathrm{~d}$ after injury. C, The graph represents $A \beta x-40$ levels normalized to protein concentration (mean $\pm S E M$ of $7 \mathrm{Gga} 3^{+/+}$and $6 \mathrm{Gga} 3^{+/-}$mice). $A \beta x-40$ levels were analyzed in mouse brain lysates using the WAKOII Human/rodent A $\beta x-40$ ELISA. A $\beta x-40$ levels are increased by $24 \%$ ( $p=$ tion factor eIF $2 \alpha$ (O'Connor et al., 2008), increased expression of a noncoding antisense BACE1 transcript (Faghihi et al., 2008), and decreased expression of the BACE1 regulating microRNAs, miR-29, and miR-107 (Hébert et al., 2008; Wang et al., 2008). Additionally, increasing evidence suggests that BACE1 is a stressinduced protease. BACE1 levels have been shown to increase in cells exposed to oxidative stress (Tamagno et al., 2002, 2003, 2005; Tong et al., 2005), apoptosis (Tesco et al., 2007), in in vivo animal models following TBI (Blasko et al., 2004), cerebral ischemia (Wen et al., 2004), and impaired energy metabolism (Velliquette et al., 2005).

In addition to the posttranslational regulation of BACE1 via caspase-mediated depletion of GGA3 and GGA1, BACE1 levels can also be regulated at the transcriptional and translational level. The BACE1 promoter contains transcription factor binding sites for NF-kB, Sp1, YY1, PPAR $\gamma, \operatorname{HIF-1} \alpha$, STAT1, and STAT3 (Christensen et al., 2004; Nowak et al., 2006; Sastre et al., 2006; Sun et al., 2006; Bourne et al., 2007; Wen et al., 2008; Cho et al., 2009). Whereas at the translational level, the phosphorylation of eIF $2 \alpha$ under conditions of energy deprivation (Velliquette et al., 2005) and the loss of microRNAs miR107 (Wang et al., 2008), miR-29a/b (Hébert et al., 2008), miR-298, and miR328 (Boissonneault et al., 2009) have been shown to increase BACE1 levels. Additionally, the BACE1 mRNA 50-untranslated region (50UTR) has been shown to act as a translational repressor (De Pietri Tonelli et al., 2004; Lammich et al., 2004; Rogers et al., 2004; Zhou and Song, 2006; Mihailovich et al., 2007).

A number of known and hypothesized BACE1-regulating factors are acutely altered (usually 3-24 $\mathrm{h}$ after injury) following experimental TBI in rodents. These

\section{$\leftarrow$}

$0.0075)$ in the injured hemisphere of $\mathrm{Gga3}^{+/-}$mice while there is no increase in the injured hemisphere of $G g a 3^{+/+}$ littermates $7 \mathrm{~d}$ after injury. $\boldsymbol{D}$, Western blot analysis of GGA3 (4167, Cell Signaling Technology) and GGA1 (H-215, Santa (ruz Biotechnology) levels in brain lysates from 6-month-old mice $7 \mathrm{~d}$ after TBI. $\boldsymbol{E}$, The graph represents GGA3 levels normalized to GAPDH (mean \pm SEM of 9 $\mathrm{Gga}^{+/+}$and $7 \mathrm{Gga}^{+/-}$mice) and expressed as arbitrary units. No difference is observed in GGA3 levels between the injured and contralateral hemispheres of $\mathrm{Gga}^{+/+}$and $\mathrm{Gga3}^{+/-}$mice. $\boldsymbol{F}$, The graph represents GGA1 levels normalized to GAPDH (mean \pm SEM of $9 \mathrm{Gga}^{+/+}$and 7 $\mathrm{Gga}^{+/-}$mice) and expressed as arbitrary units. No difference is observed in GGA1 levels between the injured and contralateral hemispheres of $\mathrm{Gga}^{+/+}$and $\mathrm{Gga}^{+/-}$mice. Statistical analysis of data performed using paired $t$ test. 
include activation/upregulation of the well known transcriptional molecules: STAT1 (Zhao et al., 2011), STAT3 (Zhao et al., 2011; Oliva et al., 2012), HIF- $1 \alpha$ (Anderson et al., 2009), NF- $\kappa \beta$ (Sanz et al., 2002), and TNF $\alpha$ (a potent activator of NF- $\kappa \beta$ pathways) (Lotocki et al., 2004). Moreover, eiF2 $\alpha$ phosphorylation is increased in the hippocampus of rodents $24 \mathrm{~h}$ after injury following fluid percussion injury (Singleton et al., 2002), while TBI induced by controlled cortical impact in rodents has been shown to acutely decrease the levels of miR-107 and miR-328 (Redell et al., 2009; Wang et al., 2010). Thus, in addition to GGA3 and GGA1 depletion, any, or all of these additional BACE1 regulatory mechanisms may also contribute to the elevation of BACE1 observed in the acute phase after injury. However, to confirm the contribution of these other mechanisms, postinjury levels of BACE1 would need to be analyzed in animals in which these molecules or pathways have been pharmacologically or genetically inhibited. At this stage, these studies are currently missing. To date this is the first study providing evidence for a molecular mechanism of BACE1 elevation following TBI taking advantage of a novel mouse model null for GGA3.

In this study, we also attempted to address the important question of how acute brain injuries (e.g., stroke and head trauma) result in chronic neurodegeneration. We have previously demonstrated that GGA3 regulates BACE1 degradation by trafficking BACE1 to the lysosomes (Tesco et al., 2007; Kang et al., 2010). Thus, GGA3 is expected to play a key role in the disposal of the BACE1 that accumulates during the acute phase after injury. Consequently, we set out to investigate the effect of GGA3 haploinsufficiency (which best resembles the depletion of GGA3 observed in AD brains) on BACE1 levels in the subacute phase of injury (7 $\mathrm{d}$ after TBI). We found that GGA3 haploinsufficiency results in sustained elevation of $\mathrm{BACE} 1$ and $\mathrm{A} \beta$ levels in the subacute phase of injury when GGA1 levels are restored.

In conclusion, our data indicate that depletion of GGA1 and GGA3, and most likely additional transcriptional and posttranscriptional mechanisms (Rossner et al., 2006; Vassar et al., 2009), engender a rapid and robust elevation of BACE1 in the acute phase after injury. However, the efficient disposal of the acutely accumulated BACE1 solely depends on GGA3 levels in the subacute phase of injury. As a consequence, impaired degradation of BACE1, e.g., because of GGA3 haploinsufficiency, represents an attractive molecular mechanism linking acute brain injury to chronic $\mathrm{A} \beta$ production and neurodegeneration. Persistent $\mathrm{A} \beta$ elevation would be predicted to result in further caspase activation and ensuing GGA1 and GGA3 depletion. According to both our previous and current findings, this would serve to further elevate BACE1 and $\mathrm{A} \beta$ levels leading to a vicious cycle in individuals affected by TBI. As such, our data strongly support the hypothesis that subjects with lower levels of GGA3 may be at increased risk to develop $\mathrm{AD}$ following acute brain injury, whether it be stroke, TBI, or some other form of major brain insult. Regulation of BACE1 levels seems to be mediated by molecular mechanisms influenced by both genetic and environmental factors. Thus, BACE1 elevation may be the first step in increasing $\mathrm{A} \beta$ and triggering $\mathrm{AD}$ pathology, at least in the sporadic cases. The identification of the molecular mechanisms that regulate BACE1 is expected to lead the discovery of novel therapeutic targets for the treatment and/or prevention of $\mathrm{AD}$.

\section{References}

Anderson J, Sandhir R, Hamilton ES, Berman NE (2009) Impaired expression of neuroprotective molecules in the HIF-1alpha pathway following traumatic brain injury in aged mice. J Neurotrauma 26:1557-1566.
Bermpohl D, You Z, Korsmeyer SJ, Moskowitz MA, Whalen MJ (2006) Traumatic brain injury in mice deficient in Bid: effects on histopathology and functional outcome. J Cereb Blood Flow Metab 26:625-633.

Blasko I, Beer R, Bigl M, Apelt J, Franz G, Rudzki D, Ransmayr G, Kampfl A, Schliebs R (2004) Experimental traumatic brain injury in rats stimulates the expression, production and activity of Alzheimer's disease betasecretase (BACE-1). J Neural Transm 111:523-536.

Bodendorf U, Danner S, Fischer F, Stefani M, Sturchler-Pierrat C, Wiederhold KH, Staufenbiel M, Paganetti P (2002) Expression of human $\beta$-secretase in the mouse brain increases the steady-state level of $\beta$-amyloid. J Neurochem 80:799-806.

Boissonneault V, Plante I, Rivest S, Provost P (2009) MicroRNA-298 and microRNA-328 regulate expression of mouse beta-amyloid precursor protein-converting enzyme 1. J Biol Chem 284:1971-1981.

Bourne KZ, Ferrari DC, Lange-Dohna C, Rossner S, Wood TG, Perez-Polo JR (2007) Differential regulation of BACE1 promoter activity by nuclear factor-kappaB in neurons and glia upon exposure to beta-amyloid peptides. J Neurosci Res 85:1194-1204.

Chen XH, Siman R, Iwata A, Meaney DF, Trojanowski JQ, Smith DH (2004) Long-term accumulation of amyloid-beta, beta-secretase, presenilin-1, and caspase- 3 in damaged axons following brain trauma. Am J Pathol 165:357-371.

Chen XH, Johnson VE, Uryu K, Trojanowski JQ, Smith DH (2009) A lack of amyloid beta plaques despite persistent accumulation of amyloid beta in axons of long-term survivors of traumatic brain injury. Brain Pathol 19:214-223.

Cho HJ, Jin SM, Son SM, Kim YW, Hwang JY, Hong HS, Mook-Jung I (2009) Constitutive JAK2/STAT1 activation regulates endogenous BACE1 expression in neurons. Biochem Biophys Res Commun 386:175-180.

Christensen MA, Zhou W, Qing H, Lehman A, Philipsen S, Song W (2004) Transcriptional regulation of BACE1, the beta-amyloid precursor protein beta-secretase, by Sp1. Mol Cell Biol 24:865-874.

Clark RS, Kochanek PM, Chen M, Watkins SC, Marion DW, Chen J, Hamilton RL, Loeffert JE, Graham SH (1999) Increases in Bcl-2 and cleavage of caspase- 1 and caspase- 3 in human brain after head injury. FASEB J 13:813-821.

Clark RS, Kochanek PM, Watkins SC, Chen M, Dixon CE, Seidberg NA, Melick J, Loeffert JE, Nathaniel PD, Jin KL, Graham SH (2000) Caspase- 3 mediated neuronal death after traumatic brain injury in rats. J Neurochem 74:740-753.

Cole SL, Vassar R (2008) The role of amyloid precursor protein processing by BACE1, the beta-secretase, in Alzheimer disease pathophysiology. J Biol Chem 283:29621-29625.

Creemers JW, Ines Dominguez D, Plets E, Serneels L, Taylor NA, Multhaup G, Craessaerts K, Annaert W, De Strooper B (2001) Processing of betasecretase by furin and other members of the proprotein convertase family. J Biol Chem 276:4211-4217.

D’Amelio M, Cavallucci V, Middei S, Marchetti C, Pacioni S, Ferri A, Diamantini A, De Zio D, Carrara P, Battistini L, Moreno S, Bacci A, Ammassari-Teule M, Marie H, Cecconi F (2011) Caspase-3 triggers early synaptic dysfunction in a mouse model of Alzheimer's disease. Nat Neurosci 14:69-76.

de Calignon A, Fox LM, Pitstick R, Carlson GA, Bacskai BJ, Spires-Jones TL, Hyman BT (2010) Caspase activation precedes and leads to tangles. Nature 464:1201-1204.

De Pietri Tonelli D, Mihailovich M, Di Cesare A, Codazzi F, Grohovaz F, Zacchetti D (2004) Translational regulation of BACE-1 expression in neuronal and nonneuronal cells. Nucleic Acids Res 32:1808-1817.

Faghihi MA, Modarresi F, Khalil AM, Wood DE, Sahagan BG, Morgan TE, Finch CE, St Laurent G 3rd, Kenny PJ, Wahlestedt C (2008) Expression of a noncoding RNA is elevated in Alzheimer's disease and drives rapid feed-forward regulation of beta-secretase. Nat Med 14:723-730.

Frohlich S, Johnson P, Moriarty J (2011) Prevalence, management and outcomes of traumatic brain injury patients admitted to an Irish intensive care unit. Ir J Med Sci 180:423-427.

Fukumoto H, Cheung BS, Hyman BT, Irizarry MC (2002) Beta-secretase protein and activity are increased in the neocortex in Alzheimer disease. Arch Neurol 59:1381-1389.

Fukumoto H, Rosene DL, Moss MB, Raju S, Hyman BT, Irizarry MC (2004) Beta-secretase activity increases with aging in human, monkey, and mouse brain. Am J Pathol 164:719-725.

Hall ED, Sullivan PG, Gibson TR, Pavel KM, Thompson BM, Scheff SW 
(2005) Spatial and temporal characteristics of neurodegeneration after controlled cortical impact in mice: more than a focal brain injury. J Neurotrauma 22:252-265.

He X, Li F, Chang WP, Tang J (2005) GGA proteins mediate the recycling pathway of memapsin 2 (BACE). J Biol Chem 280:11696-11703.

Hébert SS, Horré K, Nicolaï L, Papadopoulou AS, Mandemakers W, Silahtaroglu AN, Kauppinen S, Delacourte A, De Strooper B (2008) Loss of microRNA cluster miR-29a/b-1 in sporadic Alzheimer's disease correlates with increased BACE1/beta-secretase expression. Proc Natl Acad Sci U S A 105:6415-6420.

Hirata-Fukae C, Sidahmed EH, Gooskens TP, Aisen PS, Dewachter I, Devijver H, Van Leuven F, Matsuoka Y (2008) Beta-site amyloid precursor protein-cleaving enzyme-1 (BACE1)-mediated changes of endogenous amyloid beta in wild-type and transgenic mice in vivo. Neurosci Lett 435:186-189.

Holsinger RM, McLean CA, Beyreuther K, Masters CL, Evin G (2002) Increased expression of the amyloid precursor beta-secretase in Alzheimer's disease. Ann Neurol 51:783-786.

Jellinger KA (2004) Head injury and dementia. Curr Opin Neurol 17: 719-723.

Johnson VE, Stewart W, Smith DH (2010) Traumatic brain injury and amyloid-beta pathology: a link to Alzheimer's disease? Nat Rev Neurosci 11:361-370.

Kang EL, Cameron AN, Piazza F, Walker KR, Tesco G (2010) Ubiquitin regulates GGA3-mediated degradation of BACE1. J Biol Chem 285:24108-24119.

Knoblach SM, Nikolaeva M, Huang X, Fan L, Krajewski S, Reed JC, Faden AI (2002) Multiple caspases are activated after traumatic brain injury: evidence for involvement in functional outcome. J Neurotrauma 19: $1155-1170$.

Koh YH, von Arnim CA, Hyman BT, Tanzi RE, Tesco G (2005) BACE is degraded via the lysosomal pathway. J Biol Chem 280:32499-32504.

Lammich S, Schöbel S, Zimmer AK, Lichtenthaler SF, Haass C (2004) Expression of the Alzheimer protease BACE1 is suppressed via its $5^{\prime}$ untranslated region. EMBO Rep 5:620-625.

LeBlanc AC (2005) The role of apoptotic pathways in Alzheimer's disease neurodegeneration and cell death. Curr Alzheimer Res 2:389-402.

Lee EB, Zhang B, Liu K, Greenbaum EA, Doms RW, Trojanowski JQ, Lee VM (2005) BACE overexpression alters the subcellular processing of APP and inhibits Abeta deposition in vivo. J Cell Biol 168:291-302.

Li R, Lindholm K, Yang LB, Yue X, Citron M, Yan R, Beach T, Sue L, Sabbagh M, Cai H, Wong P, Price D, Shen Y (2004) Amyloid beta peptide load is correlated with increased beta-secretase activity in sporadic Alzheimer's disease patients. Proc Natl Acad Sci U S A 101:3632-3637.

Loane DJ, Pocivavsek A, Moussa CE, Thompson R, Matsuoka Y, Faden AI, Rebeck GW, Burns MP (2009) Amyloid precursor protein secretases as therapeutic targets for traumatic brain injury. Nat Med 15:377-379.

Lotocki G, Alonso OF, Dietrich WD, Keane RW (2004) Tumor necrosis factor receptor 1 and its signaling intermediates are recruited to lipid rafts in the traumatized brain. J Neurosci 24:11010-11016.

Luo Y, Bolon B, Kahn S, Bennett BD, Babu-Khan S, Denis P, Fan W, Kha H, Zhang J, Gong Y, Martin L, Louis JC, Yan Q, Richards WG, Citron M, Vassar R (2001) Mice deficient in BACE1, the Alzheimer's betasecretase, have normal phenotype and abolished beta-amyloid generation. Nat Neurosci 4:231-232.

McConlogue L, Buttini M, Anderson JP, Brigham EF, Chen KS, Freedman SB, Games D, Johnson-Wood K, Lee M, Zeller M, Liu W, Motter R, Sinha S (2007) Partial reduction of BACE1 has dramatic effects on Alzheimer plaque and synaptic pathology in APP transgenic mice. J Biol Chem 282:26326-26334.

Mihailovich M, Thermann R, Grohovaz F, Hentze MW, Zacchetti D (2007) Complex translational regulation of BACE1 involves upstream AUGs and stimulatory elements within the $5^{\prime}$ untranslated region. Nucleic Acids Res 35:2975-2985.

Ninan I, Arancio O (2004) Presynaptic CaMKII is necessary for synaptic plasticity in cultured hippocampal neurons. Neuron 42:129-141.

Nowak K, Lange-Dohna C, Zeitschel U, Günther A, Lüscher B, Robitzki A, Perez-Polo R, Rossner S (2006) The transcription factor Yin Yang 1 is an activator of BACE1 expression. J Neurochem 96:1696-1707.

O'Connor T, Sadleir KR, Maus E, Velliquette RA, Zhao J, Cole SL, Eimer WA, Hitt B, Bembinster LA, Lammich S, Lichtenthaler SF, Hébert SS, De Strooper B, Haass C, Bennett DA, Vassar R (2008) Phosphorylation of the translation initiation factor eIF2alpha increases BACE1 levels and promotes amyloidogenesis. Neuron 60:988-1009.

Oliva AA Jr, Kang Y, Sanchez-Molano J, Furones C, Atkins CM (2012) STAT3 signaling after traumatic brain injury. J Neurochem 120:710-720.

Redell JB, Liu Y, Dash PK (2009) Traumatic brain injury alters expression of hippocampal microRNAs: potential regulators of multiple pathophysiological processes. J Neurosci Res 87:1435-1448.

Reitz C, Brayne C, Mayeux R (2011) Epidemiology of Alzheimer disease. Nat Rev Neurol 7:137-152.

Rockenstein E, Mante M, Alford M, Adame A, Crews L, Hashimoto M, Esposito L, Mucke L, Masliah E (2005) High $\beta$-secretase activity elicits neurodegeneration in transgenic mice despite reductions in amyloid-beta levels. Implications for the treatment of Alzheimer disease. J Biochem 280:32957-32967.

Rogaeva E, Meng Y, Lee JH, Gu Y, Kawarai T, Zou F, Katayama T, Baldwin CT, Cheng R, Hasegawa H, Chen F, Shibata N, Lunetta KL, PardossiPiquard R, Bohm C, Wakutani Y, Cupples LA, Cuenco KT, Green RC, Pinessi L, et al. (2007) The neuronal sortilin-related receptor SORL1 is genetically associated with Alzheimer disease. Nat Genet 39:168-177.

Rogers GW Jr, Edelman GM, Mauro VP (2004) Differential utilization of upstream AUGs in the beta-secretase mRNA suggests that a shunting mechanism regulates translation. Proc Natl Acad Sci USA 101: 2794-2799.

Rossner S, Sastre M, Bourne K, Lichtenthaler SF (2006) Transcriptional and translational regulation of BACE1 expression-implications for Alzheimer's disease. Prog Neurobiol 79:95-111.

Sanz O, Acarin L, González B, Castellano B (2002) NF-kappaB and IkappaBalpha expression following traumatic brain injury to the immature rat brain. J Neurosci Res 67:772-780.

Sastre M, Dewachter I, Rossner S, Bogdanovic N, Rosen E, Borghgraef P, Evert BO, Dumitrescu-Ozimek L, Thal DR, Landreth G, Walter J, Klockgether T, van Leuven F, Heneka MT (2006) Nonsteroidal antiinflammatory drugs repress beta-secretase gene promoter activity by the activation of PPARgamma. Proc Natl Acad Sci U S A 103:443-448.

Sena-Esteves M, Tebbets JC, Steffens S, Crombleholme T, Flake AW (2004) Optimized large-scale production of high titer lentivirus vector pseudotypes. J Virol Methods 122:131-139.

Singleton RH, Zhu J, Stone JR, Povlishock JT (2002) Traumatically induced axotomy adjacent to the soma does not result in acute neuronal death. J Neurosci 22:791-802.

Stryke D, Kawamoto M, Huang CC, Johns SJ, King LA, Harper CA, Meng EC, Lee RE, Yee A, L'Italien L, Chuang PT, Young SG, Skarnes WC, Babbitt PC, Ferrin TE (2003) BayGenomics: a resource of insertional mutations in mouse embryonic stem cells. Nucleic Acids Res 31:278-281.

Sun X, He G, Qing H, Zhou W, Dobie F, Cai F, Staufenbiel M, Huang LE, Song W (2006) Hypoxia facilitates Alzheimer's disease pathogenesis by up-regulating BACE1 gene expression. Proc Natl Acad Sci U S A 103:18727-18732.

Tamagno E, Bardini P, Obbili A, Vitali A, Borghi R, Zaccheo D, Pronzato MA, Danni O, Smith MA, Perry G, Tabaton M (2002) Oxidative stress increases expression and activity of BACE in NT2 neurons. Neurobiol Dis 10:279-288.

Tamagno E, Guglielmotto M, Bardini P, Santoro G, Davit A, Di Simone D, Danni O, Tabaton M (2003) Dehydroepiandrosterone reduces expression and activity of BACE in NT2 neurons exposed to oxidative stress. Neurobiol Dis 14:291-301.

Tamagno E, Parola M, Bardini P, Piccini A, Borghi R, Guglielmotto M, Santoro G, Davit A, Danni O, Smith MA, Perry G, Tabaton M (2005) Betasite APP cleaving enzyme up-regulation induced by 4-hydroxynonenal is mediated by stress-activated protein kinases pathways. J Neurochem 92:628-636.

Tesco G, Koh YH, Tanzi RE (2003) Caspase activation increases betaamyloid generation independently of caspase cleavage of the betaamyloid precursor protein (APP). J Biol Chem 278:46074-46080.

Tesco G, Koh YH, Kang EL, Cameron AN, Das S, Sena-Esteves M, Hiltunen M, Yang SH, Zhong Z, Shen Y, Simpkins JW, Tanzi RE (2007) Depletion of GGA3 stabilizes BACE and enhances beta-secretase activity. Neuron 54:721-737.

Tong Y, Zhou W, Fung V, Christensen MA, Qing H, Sun X, Song W (2005) Oxidative stress potentiates BACE1 gene expression and Abeta generation. J Neural Transm 112:455-469.

Tyler SJ, Dawbarn D, Wilcock GK, Allen SJ (2002) alpha- and beta-secre- 
tase: profound changes in Alzheimer's disease. Biochem Biophys Res Commun 299:373-376.

Vassar R, Bennett BD, Babu-Khan S, Kahn S, Mendiaz EA, Denis P, Teplow DB, Ross S, Amarante P, Loeloff R, Luo Y, Fisher S, Fuller J, Edenson S, Lile J, Jarosinski MA, Biere AL, Curran E, Burgess T, Louis JC, et al. (1999) Beta-secretase cleavage of Alzheimer's amyloid precursor protein by the transmembrane aspartic protease BACE. Science 286:735-741.

Vassar R, Kovacs DM, Yan R, Wong PC (2009) The beta-secretase enzyme BACE in health and Alzheimer's disease: regulation, cell biology, function, and therapeutic potential. J Neurosci 29:12787-12794.

Velliquette RA, O'Connor T, Vassar R (2005) Energy inhibition elevates $\beta$-secretase levels and activity and is potentially amyloidogenic in APP transgenic mice: possible early events in Alzheimer's disease pathogenesis. J Neurosci 25:10874-10883.

von Arnim CA, Spoelgen R, Peltan ID, Deng M, Courchesne S, Koker M, Matsui T, Kowa H, Lichtenthaler SF, Irizarry MC, Hyman BT (2006) GGA1 acts as a spatial switch altering amyloid precursor protein trafficking and processing. J Neurosci 26:9913-9922.

Wahle T, Prager K, Raffler N, Haass C, Famulok M, Walter J (2005) GGA proteins regulate retrograde transport of BACE1 from endosomes to the trans-Golgi network. Mol Cell Neurosci 29:453-461.

Wahle T, Thal DR, Sastre M, Rentmeister A, Bogdanovic N, Famulok M, Heneka MT, Walter J (2006) GGA1 is expressed in the human brain and affects the generation of amyloid beta-peptide. J Neurosci 26: $12838-12846$

Wang WX, Rajeev BW, Stromberg AJ, Ren N, Tang G, Huang Q, Rigoutsos I, Nelson PT (2008) The expression of microRNA miR-107 decreases early in Alzheimer's disease and may accelerate disease progression through regulation of beta-site amyloid precursor protein-cleaving enzyme 1. J Neurosci 28:1213-1223.

Wang WX, Wilfred BR, Madathil SK, Tang G, Hu Y, Dimayuga J, Stromberg AJ, Huang Q, Saatman KE, Nelson PT (2010) miR-107 regulates granulin/progranulin with implications for traumatic brain injury and neurodegenerative disease. Am J Pathol 177:334-345.

Wang X, Jung J, Asahi M, Chwang W, Russo L, Moskowitz MA, Dixon CE, Fini ME, Lo EH (2000) Effects of matrix metalloproteinase-9 gene knock-out on morphological and motor outcomes after traumatic brain injury. J Neurosci 20:7037-7042.

Wen Y, Onyewuchi O, Yang S, Liu R, Simpkins JW (2004) Increased betasecretase activity and expression in rats following transient cerebral ischemia. Brain Res 1009:1-8.

Wen Y, Yu WH, Maloney B, Bailey J, Ma J, Marié I, Maurin T, Wang L, Figueroa H, Herman M, Krishnamurthy P, Liu L, Planel E, Lau LF, Lahiri DK, Duff K (2008) Transcriptional regulation of beta-secretase by p25/ cdk5 leads to enhanced amyloidogenic processing. Neuron 57:680-690.

Yang LB, Lindholm K, Yan R, Citron M, Xia W, Yang XL, Beach T, Sue L, Wong P, Price D, Li R, Shen Y (2003) Elevated beta-secretase expression and enzymatic activity detected in sporadic Alzheimer disease. Nat Med 9:3-4.

Zhao JB, Zhang Y, Li GZ, Su XF, Hang CH (2011) Activation of JAK2/STAT pathway in cerebral cortex after experimental traumatic brain injury of rats. Neurosci Lett 498:147-152.

Zhou W, Song W (2006) Leaky scanning and reinitiation regulate BACE1 gene expression. Mol Cell Biol 26:3353-3364. 